\title{
Chronic epithelial kidney injury molecule-1 expression causes murine kidney fibrosis
}

\author{
Benjamin D. Humphreys, ${ }^{1,2}$ Fengfeng Xu, ${ }^{1}$ Venkata Sabbisetti, ${ }^{1}$ Ivica Grgic, $, 1,3$ \\ Said Movahedi Naini,, ${ }^{1}$ Ningning Wang, ${ }^{1,4}$ Guochun Chen,, ${ }^{1,5}$ Sheng Xiao, ${ }^{6}$ Dhruti Patel, ${ }^{1}$ \\ Joel M. Henderson, ${ }^{7}$ Takaharu Ichimura, ${ }^{1}$ Shan Mou, ${ }^{1,8}$ Savuth Soeung, ${ }^{1}$ \\ Andrew P. McMahon, ${ }^{2,9,10,11}$ Vijay K. Kuchroo, ${ }^{6}$ and Joseph V. Bonventre ${ }^{1,2}$
}

\begin{abstract}
${ }^{1}$ Renal Division, Brigham and Women's Hospital, Department of Medicine, Harvard Medical School, Boston, Massachusetts, USA. ${ }^{2}$ Harvard Stem Cell Institute, Cambridge, Massachusetts, USA. ${ }^{3}$ Department of Internal Medicine and Nephrology, Philipps University, Marburg, Germany. ${ }^{4}$ Department of Internal Medicine and Nephrology, The First Affiliated Hospital with Nanjing Medical University, Nanjing, People's Republic of China. ${ }^{5}$ Division of Nephrology, Second Xiangya Hospital, Central South University, Changsha, Hunan, People's Republic of China. ${ }^{6}$ Center for Neurologic Disease, Brigham and Women's Hospital, Harvard Medical School, Boston, Massachusetts, USA. 'Department of Pathology and Laboratory Medicine, Boston University School of Medicine, Boston, Massachusetts, USA. ${ }^{8}$ Renal Division, Renji Hospital, Shanghai JiaoTong University School of Medicine, Shanghai, People's Republic of China. ${ }^{9}$ Department of Stem Cell and Regenerative Biology, Harvard University,

Cambridge, Massachusetts, USA. ${ }^{10}$ Department of Molecular and Cellular Biology, Harvard University, Cambridge, Massachusetts, USA.

11Department of Stem Cell Biology and Regenerative Medicine, Keck School of Medicine of the University of Southern California, Los Angeles, California, USA.
\end{abstract}

\begin{abstract}
Acute kidney injury predisposes patients to the development of both chronic kidney disease and end-stage renal failure, but the molecular details underlying this important clinical association remain obscure. We report that kidney injury molecule-1 (KIM-1), an epithelial phosphatidylserine receptor expressed transiently after acute injury and chronically in fibrotic renal disease, promotes kidney fibrosis. Conditional expression of KIM-1 in renal epithelial cells (Kim $\left.1^{R E C t g}\right)$ in the absence of an injury stimulus resulted in focal epithelial vacuolization at birth, but otherwise normal tubule histology and kidney function. By 4 weeks of age, Kim ${ }^{\text {RECtg }}$ mice developed spontaneous and progressive interstitial kidney inflammation with fibrosis, leading to renal failure with anemia, proteinuria, hyperphosphatemia, hypertension, cardiac hypertrophy, and death, analogous to progressive kidney disease in humans. Kim1 ${ }^{\text {RECtg }}$ kidneys had elevated expression of proinflammatory monocyte chemotactic protein-1 (MCP-1) at early time points. Heterologous expression of KIM-1 in an immortalized proximal tubule cell line triggered MCP-1 secretion and increased MCP-1-dependent macrophage chemotaxis. In mice expressing a mutant, truncated KIM-1 polypeptide, experimental kidney fibrosis was ameliorated with reduced levels of MCP-1, consistent with a profibrotic role for native KIM-1. Thus, sustained KIM-1 expression promotes kidney fibrosis and provides a link between acute and recurrent injury with progressive chronic kidney disease.
\end{abstract}

\section{Introduction}

Acute kidney injury (AKI) is characterized by a rapid decline in kidney function, often triggered by an ischemic or toxic insult. This clinical syndrome is associated with substantial short-term morbidity, mortality, and cost, but it had previously been assumed that patients surviving the episode made a full renal recovery (1). However, AKI is now appreciated to be markedly associated with increased risk of future chronic kidney disease (CKD), end-stage renal disease (ESRD) $(2,3)$, and long-term mortality (4). The population rate of AKI is increasing at greater than $7 \%$ per year $(5,6)$, and some estimates indicate that the incidence of AKI-related ESRD is equal to the incidence of ESRD from diabetes (7). The mechanisms that might explain the link between AKI and future CKD/ESRD are poorly understood, but peritubular capillary loss, a known consequence of AKI (8), is proposed to lead to chronic hypoxia and later development of tubulointerstitial fibrosis and $\operatorname{CKD}(9,10)$. How chronic ischemia might trigger parenchymal loss at a molecular level is unresolved.

Kidney injury molecule-1 (KIM-1), originally identified as hepatitis A virus receptor (HAVCR1, also known as Tim-1), is a type 1

Conflict of interest: Joseph V. Bonventre is an inventor on KIM-1 patents, which have been licensed by Partners Healthcare to Johnson \& Johnson, Genzyme, Biogen Idec, and other companies.

Citation for this article: J Clin Invest. 2013;123(9):4023-4035. doi:10.1172/JCI45361. transmembrane protein strongly induced by ischemic and toxic insults to kidney. It also plays diverse roles in T and B cell biology (11). In healthy kidney, KIM-1 is undetectable, but after injury, it is induced more than any other protein, in which case it localizes to the apical surface of surviving proximal tubule epithelial cells (12). The extracellular KIM-1 Ig variable domain binds and internalizes oxidized lipid as well as phosphatidylserine exposed on the outer leaflet of luminal apoptotic cells $(13,14)$, thereby aiding in nephron repair and tissue remodeling through phagocytosis of cells and debris (15). KIM-1 is expressed in CKD (16-20) where it colocalizes with areas of fibrosis and inflammation (21), and its expression correlates directly with interstitial fibrosis in human allografts (22). Increased urinary KIM-1 is an independent predictor of long-term renal graft loss and is also elevated in human nondiabetic, proteinuric CKD $(23,24)$. The expression of KIM-1 in chronic and progressive kidney disease, settings without significant numbers of apoptotic cells in the tubule lumen, the epidemiologic association of AKI with future CKD (25), and the temporal and spatial association of KIM-1 with inflammation and fibrosis suggest that it might play a pathogenic role in linking AKI to CKD and renal fibrosis.

In this study, we examined the functional consequences of chronic KIM-1 expression in renal epithelial cells. To dissociate the effects of KIM-1 expression from the pleiotropic effects of 

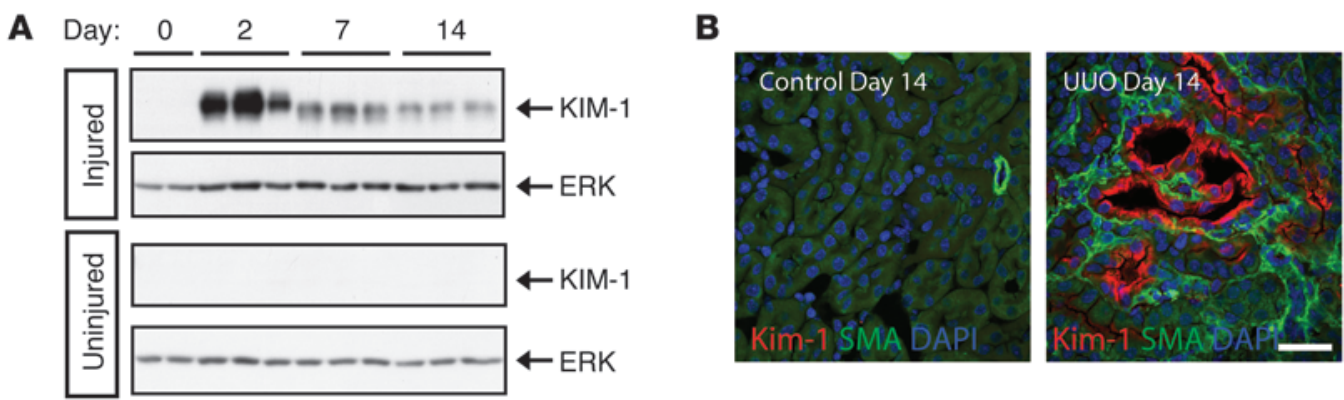

\section{Figure 1}

$\mathrm{KIM}-1$ is highly induced in fibrotic kidney injury adjacent to interstitial myofibroblasts. (A) KIM-1 protein is highly induced by 2 days after ureteral ligation, with persistent expression at days 7 and 14 as assessed by Western blot. (B) Tubular KIM-1 expression in the UUO renal fibrosis model. After fibrotic injury, KIM-1-positive epithelia are adjacent to SMA-positive interstitial myofibroblasts. Scale bar: $10 \mu \mathrm{m}$.

ischemic kidney injury used to induce KIM-1, we created a genetic model in which KIM-1 is expressed chronically in the absence of any injury stimulus. Using this model, we demonstrate here that chronic KIM-1 expression leads to inflammation, tubulointerstitial fibrosis characterized by elevated monocyte chemotactic protein-1 (MCP-1) levels and a murine CKD phenotype. In contrast, mice with mutant endogenous KIM-1 were protected from fibrosis in a mouse model of CKD and had a reduced level of MCP-1. Together, these results indicate that persistent KIM-1 expression after AKI promotes interstitial fibrosis and correlates with MCP-1 expression and further suggest that KIM-1 may represent a novel therapeutic target in CKD (26). The mouse model we have developed also recapitulates the renal and extrarenal manifestations of CKD seen in humans. These studies provide insight into how recurrent tubular injury, as reflected by persistent KIM-1 expression, might facilitate progressive CKD and lead to ESRD.

\section{Results}

To determine the kinetics of KIM-1 induction during fibrotic disease, we examined the time course for KIM-1 expression in a rodent model of renal fibrosis, unilateral ureteral obstruction (UUO). KIM-1 protein was strongly upregulated 2 days after ureteral obstruction and fell thereafter, but remained significantly elevated at day 14 (Figure 1A). KIM-1 was expressed on the apical aspect of proximal tubule epithelia, in tubules surrounded by expanded interstitium with abundant interstitial smooth muscle actin-positive myofibroblasts (Figure 1B). To distinguish between KIM-1 expression as a cause or consequence of epithelial injury and fibrosis in vivo, we created a conditional $Z / K i m 1-A P$ transgene enabling Cre recombinase-dependent activation of KIM-1 and alkaline phosphatase (AP) expression (Figure 2, A-E). Crossing the Z/Kim1-AP mouse with Six2-GFPCre mice (hereafter referred to as Six2-GC) (27), generated bigenic Kim $1^{\text {RECtg }}$ (Kim1 renal epithelial cell transgenic) mice with KIM-1 and AP expression in metanephric mesenchyme-derived kidney epithelia. Kim $1^{\text {RECtg }}$ kidneys expressed the Z/Kim1-AP transgene primarily in cortical and outer medullary epithelia, with rare transgene expression in inner medulla (Figure 2E and Supplemental Figure 1; supplemental material available online with this article; doi:10.1172/ JCI45361DS1). Mosaic transgene activity was observed with $10 \%-20 \%$ of renal tubules positive for AP activity, with a similar fraction of positive podocytes. Note, however, that despite the AP expression pattern, KIM-1 protein was only seen in tubules and never in podocytes (Figure 3C and data not shown). A comparison of the distribution of endogenous KIM-1 after UUO versus AP expression in Kim1 $1^{\text {RECtg }}$ kidneys is presented in Table 1.

Kim $1^{\text {RECtg }}$ mice were born at expected Mendelian ratios and expressed Kim1 mRNA at birth (Figure 3A). KIM-1 protein was properly sorted to the apical membrane of cortical proximal tubule epithelia (Figure 3, B and C). There was no difference in the birth weights of transgenic versus littermate control mice $(n=3$ Kim $1^{\text {RECtg }}$ or 7 littermate controls), but Kim $1^{\text {RECtg }}$ mice did not gain weight as quickly as littermate controls (Supplemental Figure 2). At birth, kidneys from Kim $1^{\text {RECtg }}$ mice were $23 \%$ smaller by weight, however, than those of littermate controls (Figure 3, D and E, $P<0.05, n=5 \mathrm{Kim} 1^{\text {RECtg }}$ or 13 control kidneys). This was associated with $43 \%$ fewer nephrons in Kim $1^{\text {RECtg }}$ kidneys without significant differences in glomerular diameter at P14 (Figure 3, F and G). Kidney histology at $\mathrm{P} 1$ showed a mild reduction in cortical thickness, with occasional microcysts that appeared to be glomerular (about $10 \%$ of total glomeruli; Figure $3 \mathrm{H}$ ) and rare large cysts (fewer than 1 per section). A detailed histologic analysis at P15 revealed normal glomeruli including foot processes, however (Figure 3, I and $\mathrm{J}$ ), as well as normal interstitium and vasculature. Focal coarse vacuolization and focal epithelial degeneration were noted only in $K i m 1^{\text {RECtg }}$ mouse kidneys ( $n=3$ Kim $1^{\text {RECtg }}$ and 3 control kidneys; Tables 2 and 3). These coarse vacuoles, suggestive of local injury, were found in about $1 \%$ of tubules (Figure 3, K and L). There were no histologic differences in other organs of Kim $1^{\text {RECtg }}$ mice when compared with organs from littermate controls (data not shown). Thus, P15 kidneys from Kim $1^{\text {RECtg }}$ mice were characterized by reduced nephron endowment and rare tubular epithelial vacuolization, but kidney histology was otherwise normal.

At 5 weeks, kidneys from $K i m 1^{R E C t g}$ mice developed a patchy mononuclear interstitial infiltrate with occasional hyaline casts and focal tubular damage. KIM-1 continued to be expressed in a subset of tubular epithelial cells along the apical membrane (Supplemental Figure 3). By 12 weeks, interstitial inflammation was extensive, together with tubular dedifferentiation, microcystic tubular dilation, hyaline casts, and fibrosis. This inflammatory, tubular injury, and fibrotic phenotype was observed in all Kim $1^{\text {RECtg }}$ mice older than 6 weeks that were examined (Figure 4A and Supplemental Figure 4). In the oldest mice, prominent periarterial inflammation was present resembling ectopic lymph nodes. Tubular injury scores confirmed these histologic observations (Figure 4B). Serum creatinine was equal between transgenic and control mice at P14, but 
A

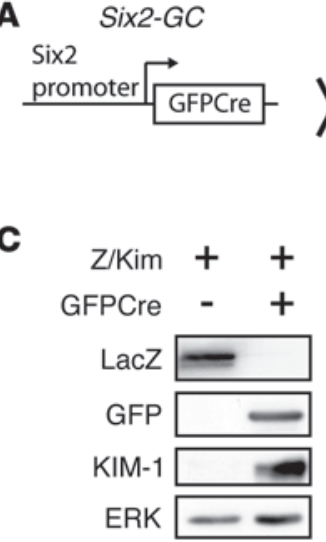

Z/Kim

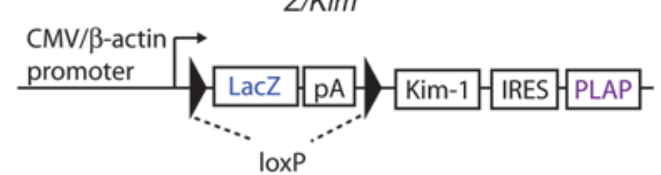

D

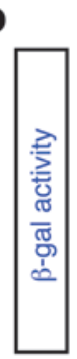

WT
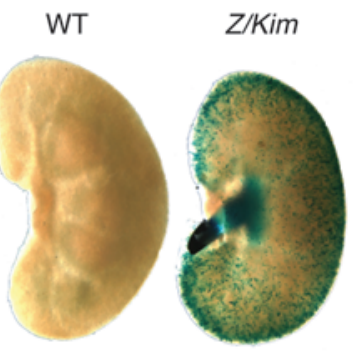

B

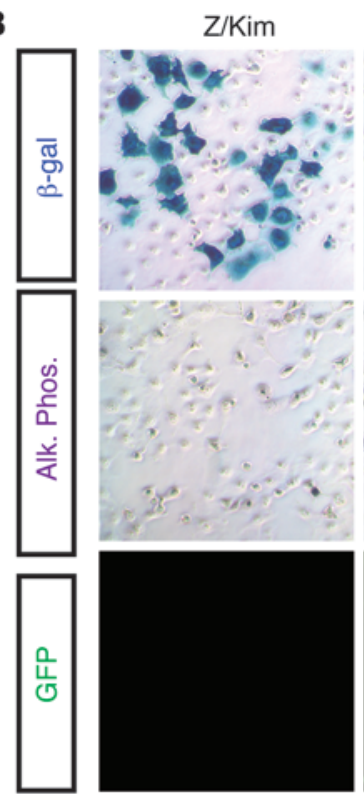

Z/Kim + GFPCre

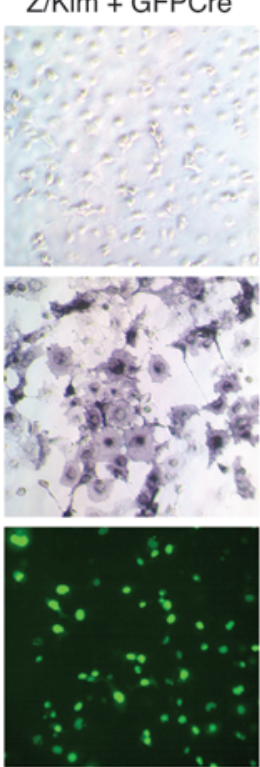

E

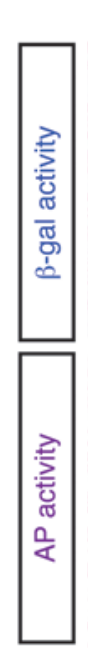

WT

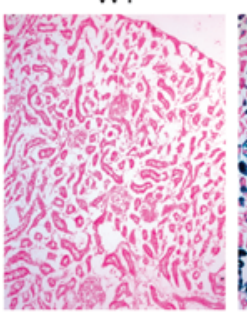

Z/Kim1AP

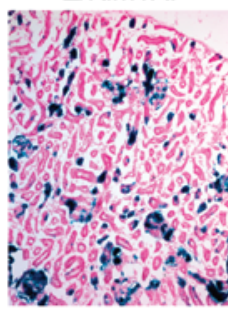

Kim1 $1^{\text {RECIg }}$

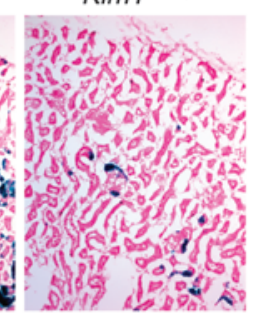

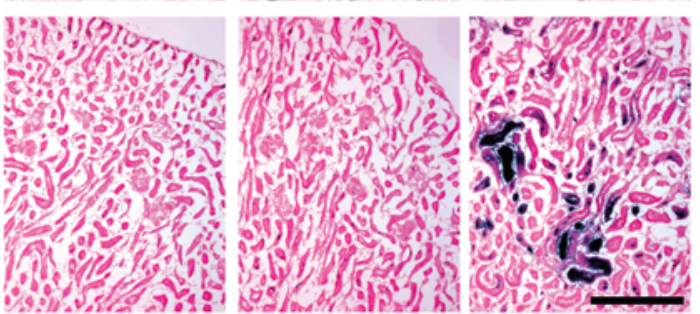

Figure 2

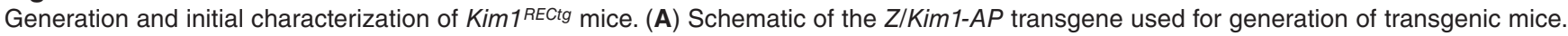
(B) Transient transfection of Cos7 cells with the Z/Kim1-AP (Z/Kim1) plasmid in the absence or presence of a plasmid directing expression of a GFPCre fusion protein verifies that LacZ is expressed before Cre-dependent recombination, and AP after. Original magnification, $\times 400$. (C) KIM-1 protein is detected only in lysates of Cos7 cells cotransfected with Z/Kim1-AP and GFPCre plasmids. (D) Wholemount X-gal stain of a Z/Kim1-AP-positive mouse and littermate control (age 4 weeks) shows mosaic LacZ activity throughout cortex. (E) WT mice express neither LacZ nor AP, whereas Z/Kim1-AP mice (age 4 weeks) exhibit mosaic LacZ expression but no AP expression. Kidney sections from bigenic Kim1RECtg mice show reduced LacZ expression and activation of AP expression in the cortex. Scale bar: $250 \mu \mathrm{m}$.

rose progressively thereafter (Figure 4C), and kidneys from aged mice were shrunken with a cobblestone appearance typical of end-

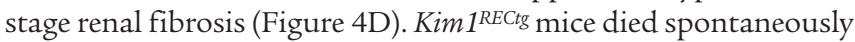
of progressive renal failure at a median age of 11 weeks (Figure 4E).

Immunohistochemistry and collagen stains confirmed early focal fibrosis surrounding isolated tubules beginning at 4 weeks, whereas older mice exhibited extensive fibrosis, with abundant $\alpha$ SMApositive interstitial myofibroblasts and collagen fiber deposition (Figure 5A). In Kim $1^{\text {RECtg }}$ mice with established fibrotic disease, AP transgene expression was expressed in some, but not all, damaged and dilated tubules, consistent with transgene expression in 10\%-20\% of tubules (Figure 5B). We did not detect any interstitial cells that expressed the AP transgene, arguing against any direct contribution of injured epithelial cells to the myofibroblast population through epithelial-to-mesenchymal transition and consistent with the notion that epithelia are not capable of contributing directly to the interstitial myofibroblast pool $(28,29)$.
Given the progressive kidney disease exhibited by Kim $1^{\text {RECtg mice, }}$ we looked for extrarenal manifestations of CKD. Cardiac hypertrophy often accompanies CKD in humans and is linked to the very high cardiac mortality associated with CKD (30). We performed cardiac ultrasound in Kim ${ }^{R E C t g}$ mice at age 10 to 12 weeks, a time when the renal phenotype is well established. At this time point, there was no increase in the systolic blood pressure (Figure 5, E-I), nor was there an increase in the interventricular septal thickness, left ventricular end-diastolic diameter, or left ventricular poster wall dimensions in Kim $1^{\text {RECtg }}$ compared with littermate controls (Table 4). There was a significantly increased percentage fractional shortening, consistent with increased cardiac contractility, which is likely a consequence of the anemia that the mice develop (Figure 5J). In contrast, Kim $1^{\text {RECtg }}$ mice that survived past 6 months of age had clear evidence of left ventricular hypertrophy, measured as ventricular wall thickness-to-diameter ratio (Figure 5, C and D). If hypertension were the primary cause of the renal fibrosis in 
A

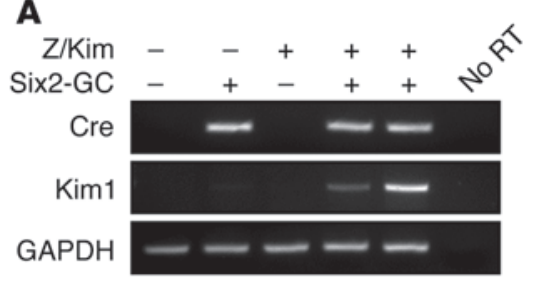

D

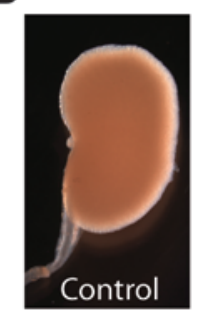

E

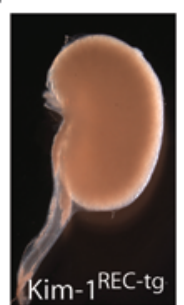

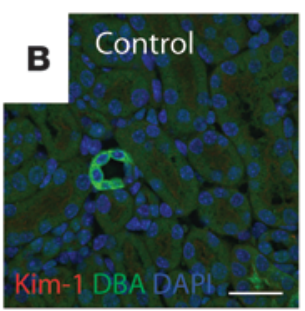

$\mathbf{F}$

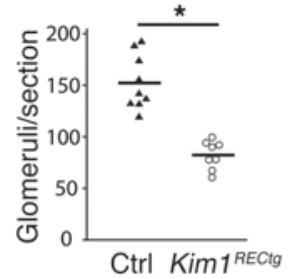

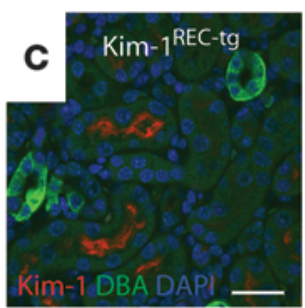

G

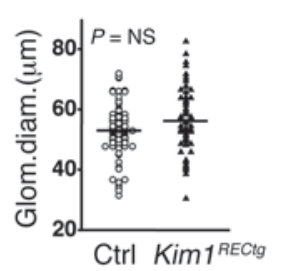

H
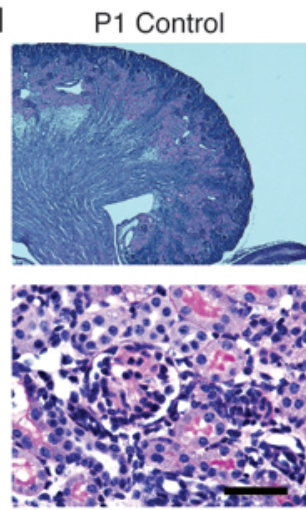

J

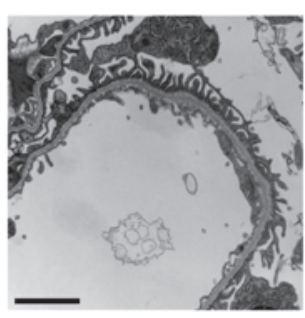

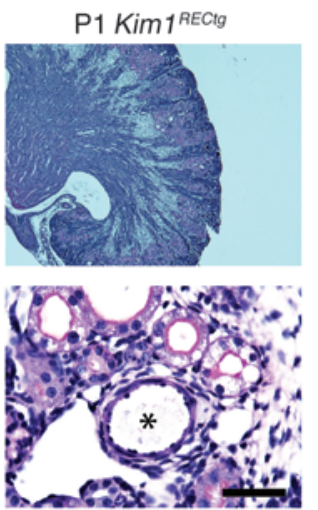

$\mathbf{K}$

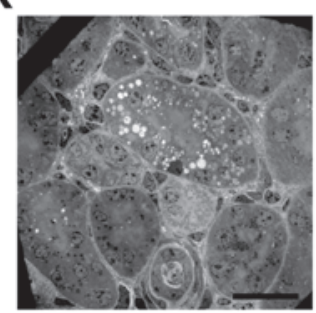

I
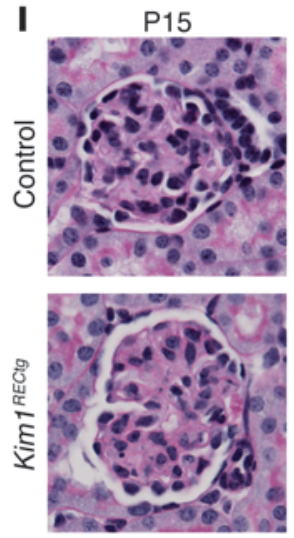

$\mathbf{L}$

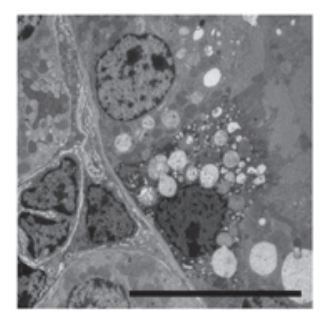

Figure 3

Tubular KIM-1 expression and phenotype of Kim1 $1^{\text {RECtg }}$ kidneys. (A) Kim1 mRNA is present in $\mathrm{P} 1$ kidneys only in bigenic

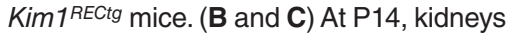
from control mice do not express KIM-1 protein, whereas kidneys from Kim1 ${ }^{R E C t g}$ mice exhibit appropriate apical expression of KIM-1 in proximal tubules. KIM-1, red; Dolichos biflorus lectin (DBA), green. Scale bar: $20 \mu \mathrm{m}$. (D and E) At birth, Kim1 ${ }^{\text {RECtg }}$ kidneys are smaller in size and $23 \%$ smaller in weight compared with those of littermate controls. (F) Kim $1^{\text {RECtg }}$ mice $(n=8)$ had $46 \%$ fewer nephrons than controls at $\mathrm{P} 14(n=9) ;{ }^{\star} P=0.0001$. (G) Glomerular diameter was not different between controls and Kim $1^{\text {RECtg }}$ mice. $(\mathbf{H})$ Low- and high-power views of $\mathrm{P} 1$ kidneys from control or Kim1 1 RECtg kidneys reveal thinned cortex and occasional cystic glomerular changes $\left(^{*}\right)$ in Kim $1^{\text {RECtg. Scale }}$ bar: $25 \mu \mathrm{m}$. (I) At P15, glomeruli of control and Kim1 ${ }^{\text {RECtg }}$ kidneys were similar, without cystic dilation. Original magnification, $\times 400$. (J) Electron microscopy of P14 Kim1 1 RECtg kidneys revealed normal glomerular architecture. Scale bar: $2 \mu \mathrm{m}$. In tubules, occasional focal coarse epithelial vacuoles were visible (K and $\mathbf{L}$ ), suggestive of epithelial injury. Scale bars: $10 \mu \mathrm{m}$.
Kim $1^{\text {RECtg }}$ mice then cardiac hypertrophy would have been expected to occur by the 10 - to 12 -week time point when renal injury and dysfunction were severe.

The presence of a normal blood pressure at 10 to 12 weeks is also consistent with the notion that hypertension was not simply a consequence of reduced nephron endowment, because it did not precede the renal phenotype, but rather that hypertension was a consequence of severe reduction in glomerular filtration rate (Figure 5E). The severe and progressive anemia developed by the Kim $1^{\text {RECtg }}$ mice (Figure 5F) was normocytic (mean corpuscular volume was not different between control and Kim $1^{\text {RECtg }}$ mice; data not shown). Late stages of CKD are characterized by hyperkalemia, hyperphosphatemia, and hypoalbuminemia. Thus, Kim $1^{\text {RECtg }}$ mice displayed all 3 of these characteristics in a progressive fashion over time (Table 5).

The phenotype is not a result of primary proteinuria, podocyte expression of KIM-1, or general toxicity of AP. Chronic proteinuria of any cause has been proposed to drive renal fibrosis. Kim $1^{\text {RECtg }}$ mice developed proteinuria after 4 weeks of age, subsequent to tubular damage and leukocyte influx (Figure 5G and Supplemental Figure 5). Importantly, there was no proteinuria in Kim $1^{\text {RECtg }}$ mice at P14, when podocyte foot processes and glomerular capillary endothelium were normal (Figure 3J). Since KIM-1 is not normally expressed in podocytes, we investigated whether the Kim $1^{\text {RECtg }}$ phenotype was

Table 1

Comparison of endogenous Kim1 expression after UUO and AP expression in Kim1 ${ }^{\text {RECtg }}$ mice

\begin{tabular}{lcccc}
\hline & Podocytes & $\begin{array}{c}\text { Proximal } \\
\text { tubule } \\
\text { Endogenous KIM-1 }\end{array}$ & $\begin{array}{c}\text { Distal } \\
\text { tubules }\end{array}$ & $\begin{array}{c}\text { Collecting } \\
\text { duct }\end{array}$ \\
Kim1RECtg (AP) & $10 \%-20 \%-100 \%$ & 0 & 0 \\
& & $10 \%-20 \%$ & $10 \%-20 \%$ & 0
\end{tabular}


Table 2

Histologic analysis of Kim1 RECtg or control kidney tubules at P15

\begin{tabular}{lccc} 
Genotype & $\begin{array}{c}\text { Tubule histology } \\
\text { Reabsorption } \\
\text { granules }\end{array}$ & Vacuolization & $\begin{array}{c}\text { Epithelial } \\
\text { degeneration }\end{array}$ \\
Control 1 & $1+$ (focal apical) & $1+$ (focal apical) & 0 \\
Control 2 & $1+$ (focal apical) & $1+$ (focal apical) & 0 \\
Control 3 & 0 & \pm (focal apical) & 0 \\
Transgenic 1 & \pm (focal) & $1+$ (focal coarse) & \pm (focal) \\
Transgenic 2 & \pm (focal) & $1+$ (focal coarse) & $1+$ (focal) \\
Transgenic 3 & $1+$ (focal apical) & $1+$ (focal coarse) & $1+$ (focal) \\
\hline
\end{tabular}

a consequence of delayed toxicity to podocytes from glomerular KIM-1 expression. Mice with KIM-1-AP expression exclusively in podocytes (bigenic Podocin-Cre;Z/Kim1-AP) had expression of AP activity in $55 \%$ of glomeruli in a focal pattern (Supplemental Figure 6) with histologically normal glomeruli (data not shown). There was no effect on kidney size, and mice did not develop proteinuria even up to 6 months of age. Serum creatinine and hematocrit have the same values as in littermate controls (Figure 5, I and J). Taken together, these observations show that renal fibrosis was not a secondary consequence of abnormal glomerular development, early hypertension, or podocyte expression of KIM-1. We also evaluated the possibility that AP expression alone in Kim $1^{\text {RECtg }}$ mice might mediate kidney damage independently of KIM-1 (31). However, bigenic Six2-GC;Z/AP mice that expressed the AP transgene (without KIM-1) in nearly $100 \%$ of renal epithelia (Supplemental Figure 5) had normal renal histology, no proteinuria, and normal serum creatinine, even in the case of aged mice (Figure 5I and data not shown).

Characterization of KIM-1-induced kidney inflammation. Since early inflammation was a prominent histologic feature in Kim $1^{\text {RECtg }}$ mice, we next sought to characterize this infiltrate at the earliest time that disease appeared, 4 weeks. There was no interstitial infiltrate at 2 weeks, but $\mathrm{CD}^{+}$lymphocytes and $\mathrm{F} 4 / 80^{+}$macrophages and dendritic cells migrated into Kim $1^{\text {RECtg }}$ kidneys by 4 weeks, and their appearance correlated with increased interstitial cell proliferation at this time point (Figure 6, A-C). KIM-1 has recently been identified as an endogenous ligand for the activating receptor leukocyte monoimmunoglobulin-like receptor 5 (LMIR5) (also known as CD300b), and binding of KIM-1 to LMIR5 promotes neutrophil influx regulated by myeloid cells (32). However, there were no neutrophils at
4 weeks in Kim1 $1^{\text {RECtg }}$ kidneys, and they were only observed at late stages of disease (Figure 6C and data not shown). $\mathrm{CD}^{+}$lymphocytes were located in a focal pattern at early stages, often adjacent to KIM-1-positive tubules (Figure 6D), suggesting that secreted factors from KIM-1-expressing cells might be responsible for recruiting inflammatory cells to the kidney in Kim $1^{\text {RECtg mice. }}$

Tubule damage is known to contribute to and be exacerbated by inflammation. We therefore tested whether tubular damage could be detected using urinary biomarkers at 4 weeks. Since KIM-1 itself undergoes proteolytic cleavage, resulting in release of the soluble ectodomain that might have the capacity to send a proinflammatory signal itself (32), we measured urinary KIM-1 levels. Even at 4 weeks, when disease was mild, urinary KIM-1 levels were higher in Kim1 $1^{\text {RECtg }}$ mice compared with controls, as expected (Figure 7A). Consistent with early epithelial damage, urinary $\mathrm{N}$-acetyl- $\beta$-D-glucosaminidase (NAG) was also increased at 4 weeks and increased further at 8 weeks (Figure 7B). We further measured the levels of cytokines and chemokines known to be activated by epithelial pattern recognition receptors that might mediate leukocyte recruitment. We detected a strong upregulation of mRNA encoding a panel of cytokines capable of being secreted by epithelial cells at 4 weeks (Figure 7C). Three of these cytokines, CXCL-1, MCP-1, and TGF- $\beta$, were upregulated at 2 weeks - a time point without evident histologic damage.

The inflammation observed in Kim $1^{\text {RECtg }}$ mice coupled with increased proinflammatory cytokine expression suggested the possibility that KIM-1 might directly regulate epithelial cytokine expression. To test this possibility, we stably expressed either vector alone (pcDNA-LLC) or KIM-1 (KIM-1-LLC) in LLC-PK1 porcine proximal tubule cells. The supernatant from these cultures was collected and assessed for cytokines. The supernatant from KIM-1-expressing, but not control, cultures showed significant elevations in the levels of TGF- $\beta$, MCP-1, and IL-6 (Figure 7, D-F).

We next asked whether conditioned medium from KIM-1-LLC cells might also induce chemotaxis. pcDNA-LLC and KIM-1-LLC cells were plated in lower wells of the Boyden chamber until confluency, then washed and incubated overnight with DMEM. $5 \times 10^{5}$ phorbol ester-activated U937 cells or primary mouse BM-derived macrophages (mBMDM) were added to the upper wells. After 3 hours, cells that migrated through the filters were fixed, stained, and quantitated. In both cases, significantly more cells migrated in response to KIM-1-LLC medium compared with control (Figure 7, $\mathrm{G}$ and $\mathrm{H}$ ). This effect could be largely abrogated by addition of a neutralizing anti-MCP-1 antibody (Figure 7I), consistent with the increased expression of MCP-1 detected in KIM-1-LLC supernatant.

Table 3

Histologic analysis of Kim1 ${ }^{R E C t g}$ or control kidney glomeruli at P15

Glomerular histology

$\begin{array}{lcc}\text { Genotype } & \begin{array}{c}\text { Podocyte } \\ \text { effacement }\end{array} & \text { Vacuolization } \\ \text { Control 1 } & \pm \text { (focal) } & 0 \\ \text { Control 2 } & \pm \text { (focal) } & 0 \\ \text { Control 3 } & \pm \text { (focal) } & 0 \\ \text { Transgenic 1 } & 0 & 0 \\ \text { Transgenic 2 } & \pm \text { (focal) } & \pm \text { (focal) } \\ \text { Transgenic 3 } & 1+\text { (focal) } & 00\end{array}$

$\begin{array}{ccc}\begin{array}{c}\text { Reabsorption } \\ \text { granules }\end{array} & \begin{array}{c}\text { GBM } \\ \text { thickening }\end{array} & \begin{array}{c}\text { Endo } \\ \text { fenestration loss }\end{array} \\ 0 & 0 & \pm \text { (focal) } \\ 0 & 0 & \pm \text { (focal) } \\ 0 & 0 & \pm(\text { focal) } \\ 0 & 0 & \pm \text { (focal) } \\ 0 & 0 & \pm \text { (focal) } \\ 0 & 0 & 1+(\text { focal) }\end{array}$

expansion
0
0
0
0
\pm (focal)

GBM, glomerular basement membrane; Mes., mesangial. 
A
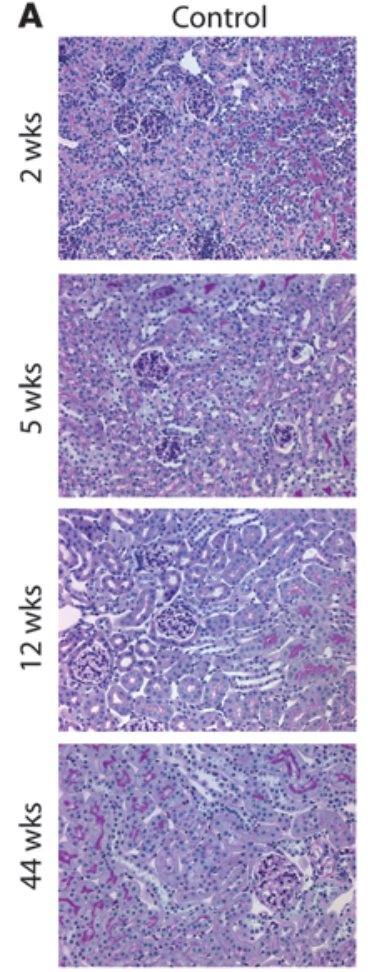

Kim1 ${ }^{\text {RECCIg }}$
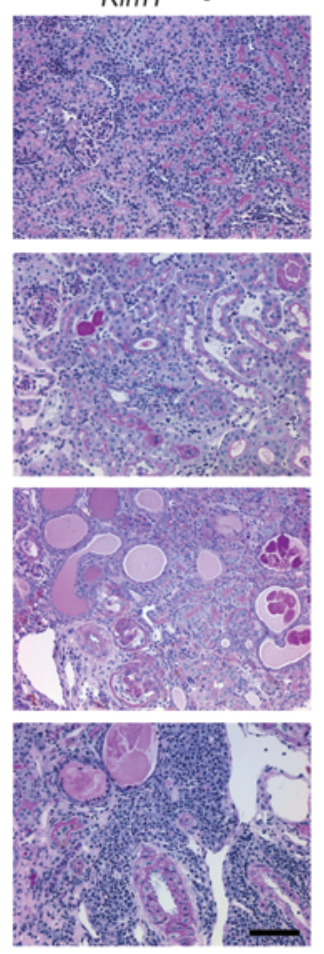

B

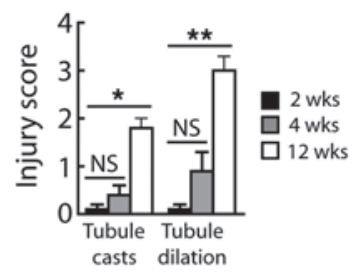

C

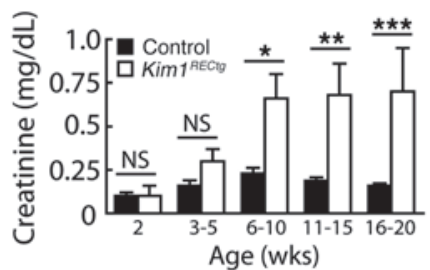

D

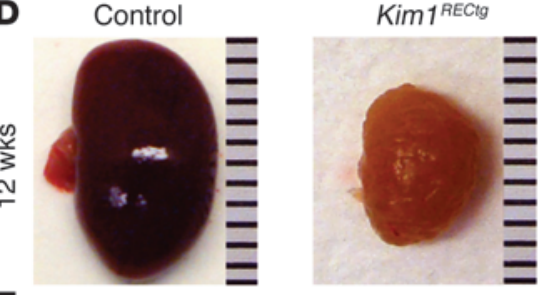

E

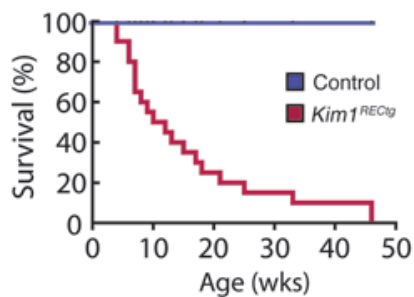

Figure 4

Spontaneous inflammation, tubule injury, renal failure, and death in Kim1RECtg mice. (A) Periodic acid-schiff stain of control or Kim1RECtg kidneys between 2 and 44 weeks of age reveals no difference in histology at 2 weeks, but progressive interstitial mononuclear cell infiltration, tubule dilation, epithelial simplification and interstitial expansion in Kim $1^{\text {RECtg }}$ kidneys only at later time points. Scale bar, $50 \mu \mathrm{m}$. (B) Tubule cast and injury scores rise with age in Kim1 $1^{\text {RECtg }}$ kidneys $(n=3)$ compared with littermate controls $(n=3) .{ }^{*} P=0.001,{ }^{\star *} P=0.0009$, NS (not significant). (C) Serum creatinine begins to rise between 3 and 5 weeks and rises progressively in Kim1 ${ }^{\text {RECtg }}$ kidneys ( $n=4-8$ per time point) compared with controls $(n=5-11$ per time point), ${ }^{\star} P=0.002$, ${ }^{\star *} P=0.006,{ }^{* * *} P=0.004$. (D) A Kim1 $1^{\text {RECtg }}$ kidney is markedly reduced in size and fibrotic at 10 weeks compared with littermate control kidney. (E) Kim1 1 RECtg mice die spontaneously at a median age of 11 weeks $(n=20$, both groups), $P<0.0001$.
Soluble fibronectin is a known inducer of proinflammatory cytokines in renal epithelial cells (33), and the KIM-1-expressing cell line had substantially elevated fibronectin expression compared with control cells. Moreover, in a third cell line that expressed a point mutant of a cytoplasmic tyrosine within a consensus phosphorylation sequence (Y350F), fibronectin levels matched those of control LLC-PK1 cells (Figure 7J).

These findings indicate that KIM-1 expression drives proinflammatory cytokine expression, suggesting a mechanism to explain enhanced leukocyte infiltration at early time points in the Kim $1^{\text {RECtg }}$ mouse model. KIM-1 also drives fibronectin expression in LLCPK1 cells through a mechanism that requires cytoplasmic tyrosine Y350, suggesting that fibronectin upregulation may underlie the proinflammatory cytokine expression observed.

KIM-1 mutant mice are protected from renal fibrosis in the UUO model. The Kim $1^{\text {RECtg }}$ phenotype suggested that mice with mutant KIM-1 might be protected from kidney fibrosis. To test this hypothesis, we analyzed mice carrying a deletion of exon 3 of the KIM-1 locus $\left(K i m 1^{\Delta m u c}\right)$, resulting in an in-frame deletion of the extracellular mucin domain. This mutation results in a smaller KIM- 1 polypeptide that is defective in KIM-1-dependent phagocytic function (34). When subjected to UUO injury, KIM-1 ${ }^{\Delta \text { muc }}$ protein was upregulated, but as expected, it migrated faster on Western blot than native KIM-1, reflecting the absence of the heavily glycosylated mucin domain encoded by exon 3 (Figure 8A).

Both control and $\operatorname{Kim} 1^{\Delta m u c}$ mice $(n=10$ each) were subjected to UUO and sacrificed on day 10 . Kim $1^{\Delta m u c}$ mice had significantly reduced interstitial collagen deposition as well as reduced tubular injury scores (Figure 8, B-D). mRNA levels of the myofibroblast marker $\alpha$ SMA were also reduced in Kim $1^{\Delta m u c}$ mice, in addition to levels of collagen $1 \alpha 1$ and fibronectin (Figure 8, E and F). Taken together, these findings indicate that $\operatorname{Kim} 1^{\Delta m u c}$ mice are protected from renal fibrosis in the UUO model. Since proinflammatory cytokine levels were increased in Kim ${ }^{\text {RECtg }}$ mice and KIM-1 expression regulates MCP-1, TGF- $\beta$, and IL- 6 secretion in vitro, we analyzed the same panel of cytokines in Kim $1^{\Delta m u c}$ kidney samples before and after UUO. MCP-1 levels were significantly reduced in Kim $1^{\Delta m u c}$ compared with control at day 10 of UUO (Figure 8H), while other cytokine mRNAs were unchanged (data not shown). This result, combined with our previous data, strongly implicates MCP-1 as a mediator of KIM-1-dependent fibrosis in the mouse kidney.

\section{Discussion}

Resident kidney epithelial cells play an important role in detection of injury, regulation of the inflammatory and tissue repair responses, and mediation of interstitial fibrosis through paracrine mechanisms $(35,36)$. In this study, we hypothesized that KIM-1 might regulate kidney inflammation and fibrosis when its expression is prolonged because: (a) it is upregulated very early after kidney injury and is thus poised to serve as a sentinel of damage; (b) it is expressed in chronic fibrosing kidney disease, where it colocalizes with areas of fibrosis and inflammation $(21,22)$; (c) it is a phosphatidylserine receptor and may function in a manner similar to that of Toll-like receptors that are known to regulate innate immu- 
A
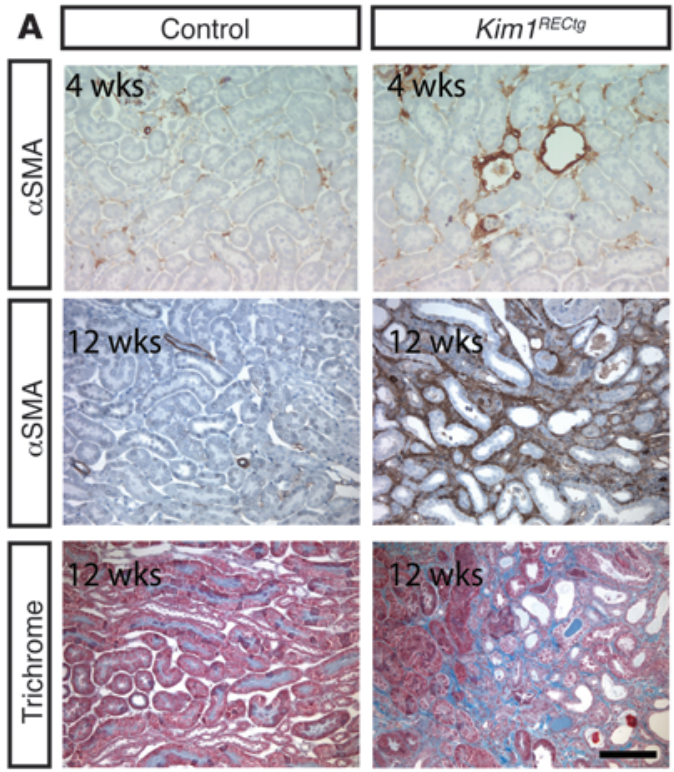
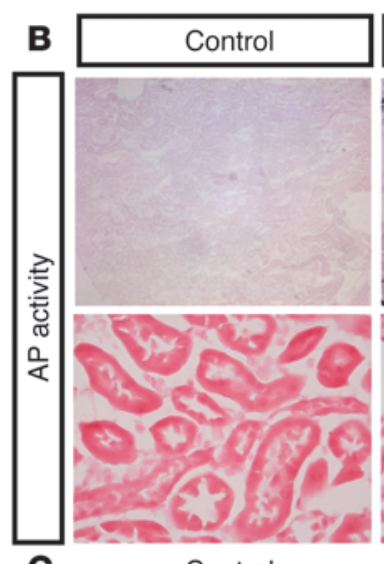

Control

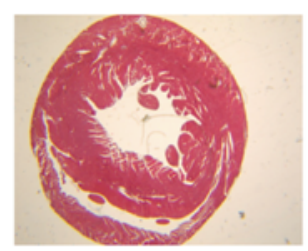

G
D

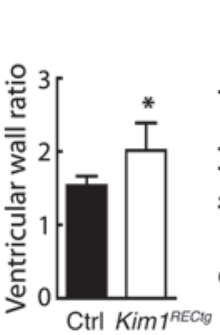

H

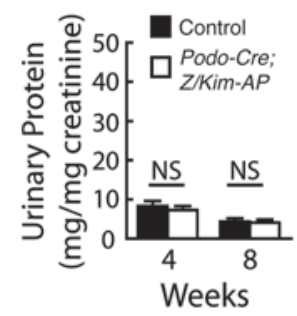

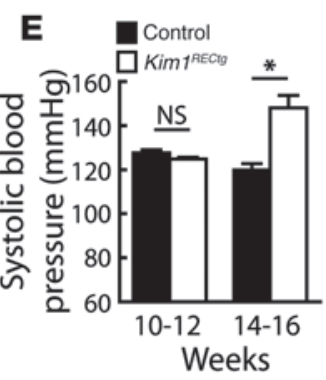

I

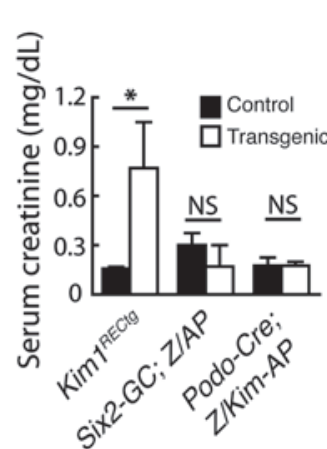

F

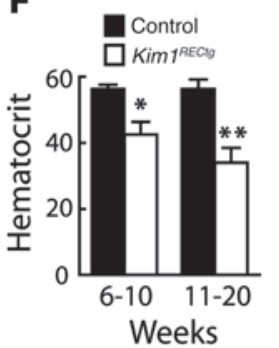

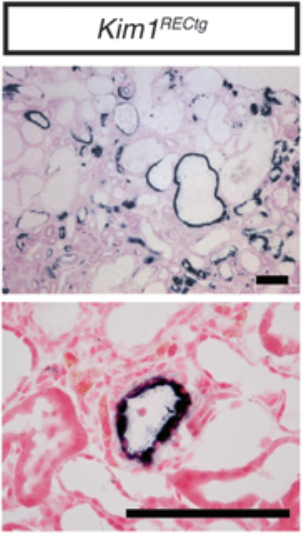

$\operatorname{Kim} 1^{\text {RECIg }}$

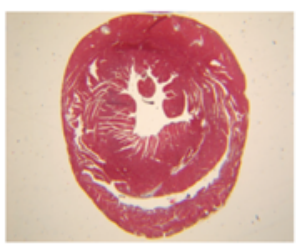

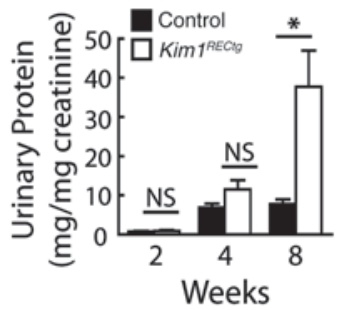

J Control

$\square$ Transgenic

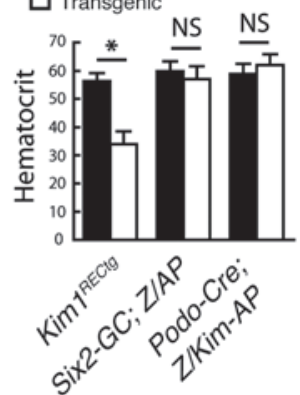

Figure 5

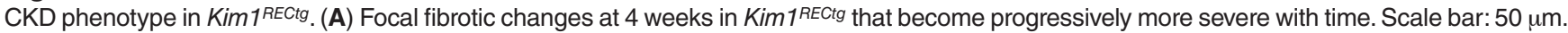
(B) AP expression identifies cells that have undergone Cre-mediated recombination. No AP-positive cells were found in fibrotic interstitium. Scale bar: $50 \mu \mathrm{m}$. (C) Concentric left ventricular hypertrophy in aged Kim1 ${ }^{R E C t g}$, trichrome stain. (D) Ventricular wall ratio (outer to inner diameter) was increased in aged (range, 12-45 weeks) $\operatorname{Kim} 1^{\text {RECtg }}\left(n=5\right.$ for each group). ${ }^{*} P=0.03$. (E) Younger $\operatorname{Kim} 1^{\text {RECtg }}(n=3)$ do not have hypertension, but older $\operatorname{Kim} 1^{\text {RECtg }}(n=5)$ do develop hypertension. ${ }^{*} P=0.0002$. (F) Hematocrit in control $(n=10)$ or $\operatorname{Kim} 1^{\text {RECtg }}(n=5)$ mice between 6 and 10 weeks or control $(n=13)$ and Kim1RECtg $(n=7)$ mice measured between 10 and 20 weeks of age. ${ }^{*} P=0.001 ;{ }^{* *} P=0.0001$. (G) Total urinary protein is elevated in 8-week-old $\operatorname{Kim} 1^{R E C t g}(n=3-5)$ but not at 2 or 4 weeks compared with littermate controls $(n=4-6)$. ${ }^{*}=0.01$. $(\mathbf{H})$ Urinary protein is not elevated in mice with expression of KIM-1 in podocytes alone at either 4 or 8 weeks. (I) Serum creatinine 13- to 20-week-old mice comparing control ( $n=12$ ) and Kim1RECtg $(n=8)$, control $(n=6)$ and Six2-GC;ZIAP $(n=7)$, or control $(n=4)$ and Podocin-Cre;Z/Kim1-AP $(n=4) .{ }^{*} P=0.006$, NS. $(J)$ Anemia was seen in Kim1 ${ }^{\text {RECtg }}$ but not control mice or mice in which KIM-1 was expressed in podocytes. Control refers to mice with neither transgene or 1 transgene for all groups. ${ }^{*} P<0.0001, \mathrm{NS}$. 
Table 4

Echocardiography in Kim1 ${ }^{\text {RECtg }}$ mice at 2.5-3 months

\begin{tabular}{lcc}
\hline & $\begin{array}{c}\text { Control } \\
(\boldsymbol{n}=7)\end{array}$ & $\begin{array}{c}\boldsymbol{K} \text { Kim1 } \\
(\boldsymbol{n}=\mathbf{3})\end{array}$ \\
Interventricular septal thickness $(\mathrm{mm})$ & $0.69 \pm 0.06$ & $0.75 \pm 0.04$ \\
Left ventricular end-diastolic diameter $(\mathrm{mm})$ & $3.40 \pm 0.33$ & $3.37 \pm 0.09$ \\
Left ventricular posterior wall thickness $(\mathrm{mm})$ & $0.76 \pm 0.05$ & $0.79 \pm 0.04$ \\
Fractional shortening (\%) & $38.73 \pm 3.55$ & $46.71 \pm 4.72^{\mathrm{A}}$ \\
\hline
\end{tabular}

AP $<0.05$.

nity (15); and (d) blockade of KIM-1 by monoclonal antibodies in other inflammatory conditions reduces disease pathology (37-40). The progressive kidney inflammation and fibrosis observed in Kim $1^{\text {RECtg }}$ mice are consistent with this hypothesis and, combined with the extrarenal manifestations described here, establish the Kim $1^{\text {RECtg }}$ mouse as what we believe to be a novel rodent model of progressive CKD and implicate the KIM-1 protein as a target for antifibrotic therapy in man (26).

The lower nephron endowment in our Kim1 $1^{\text {RECtg }}$ mouse model most likely reflects activation of KIM-1 expression in metanephric mesenchyme with effects on kidney development. Low nephron number is a recognized risk factor for the development of hypertension and possibly CKD (41). However, reduction in nephron number by $28 \%-40 \%$ results in no, or very mild, interstitial fibrosis, even at late time points of greater than 1 year in a number of models (42-45). In contrast, Kim $1^{\text {RECtg }}$ mice described here had a comparable degree of nephron reduction (43\%), but all developed spontaneous fibrosis starting at 4 weeks of age, with progressive renal insufficiency, proteinuria, renal fibrosis, and death at a median age of 19 weeks. Further arguing against a direct role of reduced nephron endowment in the observed phenotype, hypertension, proteinuria, and cardiac hypertrophy did not develop until well after the onset of renal fibrosis, and directed expression of the Z/Kim1-AP transgene in podocytes alone had no phenotype.

The primary finding of the current report is that chronic KIM-1 expression in renal epithelial cells directly causes interstitial inflammation followed by progressive fibrotic renal disease. Our data suggest that epithelial cells that express KIM-1 chronically may act in a paracrine fashion to recruit mononuclear cells to the renal interstitium, setting up a proinflammatory cascade, ultimately leading to further tubule damage and loss of renal function. We observed an early influx of leukocytes in the Kim $1^{\text {RECtg }}$ mouse model associated with early elevation in the proinflammatory cytokine MCP-1, a potent cytokine that mediates mononuclear cell recruitment and parenchymal cell activation. In contrast, in the Kim $1^{\Delta m u c}$ mouse model, in which KIM-1-induced phagocytosis is impaired, we observed reduced levels of MCP-1 and a reduction in renal fibrosis. Finally, our observation that stable expression of KIM-1 in proximal tubule cells in vitro triggers MCP-1 release and macrophage chemotaxis is consistent with a model in which chronic epithelial KIM-1 expression causes epithelial MCP-1 release, triggering leukocyte influx and ultimately kidney fibrosis. The results provided do not offer proof that MCP-1 is most important in triggering leukocyte influx, though an important role for MCP-1 is supported by the observation that mice deficient in the major MCP-1 receptor, CCR2, are protected from renal fibrosis (46).

The recent finding that soluble KIM-1 ectodomain serves as a ligand for the LMIR5/CD300b-activating receptor on resident macrophages provides indirect support for a model in which KIM-1 regulates inflammation (32); however, it is unclear whether soluble KIM- 1 is required in our model, since we observed proinflammatory cytokine secretion from epithelia themselves. Similarly, further investigation is required to determine whether ligation of KIM-1 by apoptotic bodies, oxidized lipid, or some other unidentified KIM-1 ligand is required for proinflammatory signaling, analogous to the requirement of Toll-like receptor ligands for induction of inflammatory responses in macrophages (47).

The stimulus for sustained KIM-1 expression after AKI or during CKD requires further investigation. The most likely explanation is that AKI itself causes peritubular capillary rarefaction (8), leading to chronic tubular hypoxia, which is a potent stimulus for KIM-1 expression (12). Chronic KIM-1 expression will promote further tubulointerstitial inflammation, capillary loss, and hypoxia, further inducing KIM-1 and creating a positive feedback loop of hypoxia and inflammation that culminate in tubulointerstitial fibrosis.

Our study suggests what we believe to be a novel role for chronic KIM-1 expression in the pathogenesis of renal fibrosis and through activation of the innate immune system and leukocyte recruitment. In contrast, very early induction of KIM-1 after AKI may serve an adaptive function to clear apoptotic and necrotic cells and debris and thereby decrease the early response of the immune system at a site of tissue damage. Persistent KIM-1 expression is perhaps maladaptive through chronic uptake of cell toxic components of the tubular lumen, thereby promoting chronic inflammation and ultimately renal fibrosis. Thus KIM-1 may represent a novel therapeutic target in fibrotic kidney disease, and antagonizing KIM-1 signaling might ameliorate renal fibrosis in CKDs.

\section{Methods}

Mouse strains. A KIM-1 cDNA was inserted into the NotI site of the $Z / A P$ plasmid (48), and linearized Z/Kim1-AP transgene was introduced into FVB zygotes (Charles River Laboratories) by pronuclear injection. Three independent founder lines were obtained; all exhibited Six2-GC-dependent transgene expression, and the line with highest outer medulla and cortex transgene expression was selected for further analysis. The Z/Kim1-AP transgenic was maintained on an FVB $\times$ C57BL/6J (Jackson Laboratory) mixed background.

Z/Kim1-AP transgenic mice were crossed with the Six2-GC Cre driver line (27) maintained on a CD- $1 \times$ Swiss Webster (Taconic) $\times$ C57BL/6J (Jackson Laboratory) mixed background. In other experiments, the Z/Kim1-AP

\section{Table 5}

Biochemical parameters in Kim1 ${ }^{\text {RECtg }}$ mice

\begin{tabular}{lcc}
\hline & Control $(\boldsymbol{n}=\mathbf{1 0})$ & $\operatorname{Kim1~}^{\text {RECtg }}(\boldsymbol{n}=\mathbf{6})$ \\
& & \\
$\mathrm{BUN}(\mathrm{mg} / \mathrm{dl})$ & $23.4 \pm 1.1$ & $99.0 \pm 25.0^{\mathrm{A}}$ \\
$\mathrm{Cr}(\mathrm{mg} / \mathrm{dl})$ & $0.12 \pm 0.01$ & $0.47 \pm 0.1^{\mathrm{A}}$ \\
$\mathrm{Na}(\mathrm{mM})$ & $149 \pm 0.9$ & $148.1 \pm 2.3$ \\
$\mathrm{~K}(\mathrm{mM})$ & $5.9 \pm 0.3$ & $7.6 \pm 0.8$ \\
$\mathrm{Cl}(\mathrm{mM})$ & $104.3 \pm 0.7$ & $101.0 \pm 1.6$ \\
Albumin & $2.9 \pm 0.1$ & $2.6 \pm 0.1$ \\
Calcium $(\mathrm{mg} / \mathrm{dl})$ & $9.2 \pm 0.1$ & $10.2 \pm 0.2^{\mathrm{A}}$ \\
Phosphorus $(\mathrm{mg} / \mathrm{dl})$ & $7.8 \pm 0.3$ & $10.14 \pm 1.0$ \\
& & \\
\hline
\end{tabular}

AP $<0.05$. 
A
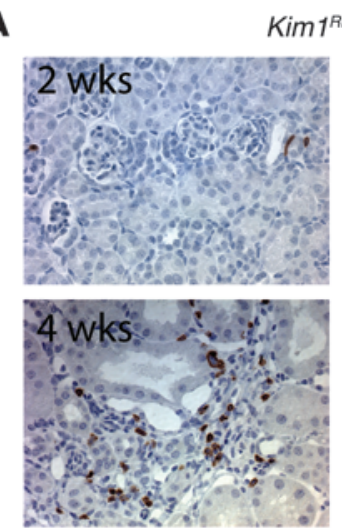

C

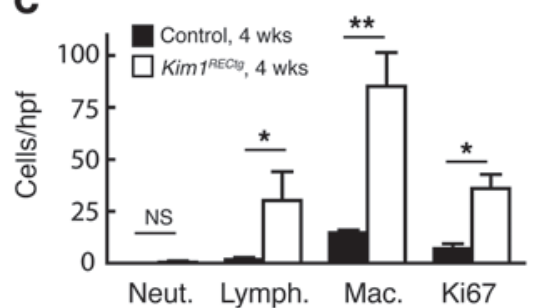

B
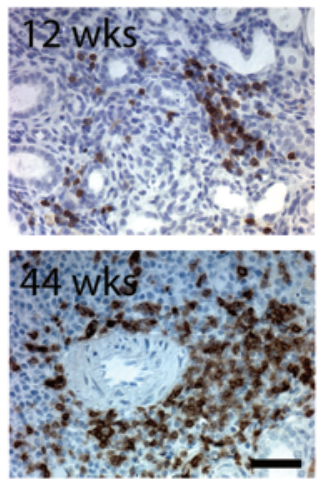

D

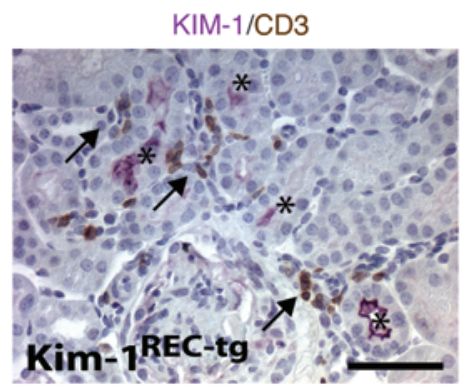

\section{Figure 6}

Leukocyte infiltration in Kim $1^{\text {RECtg }}$. (A) CD3 $3^{+}$lymphocyte infiltration begins at 4 weeks in Kim $1^{\text {RECtg }}$ and is observed in tubulointerstitium, especially surrounding vessels in older animals. Scale bar: $50 \mu \mathrm{m}$. (B) F4/80-positive peritubular macrophages and dendritic cells are increased at 4 weeks

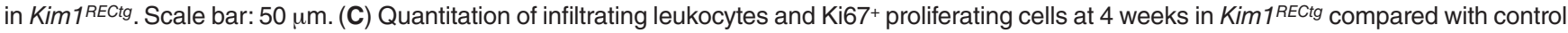
kidneys ( $n=3$ for each). ${ }^{*} P<0.0001 ;{ }^{\star \star} P=0.0003$. Neut., neutrophil; Lymph., lymphocyte; Mac., macrophage. (D) CD3-positive lymphocytes (brown) are frequently identified adjacent to KIM-1-positive (purple, ${ }^{*}$ ) tubules. Scale bar: $25 \mu \mathrm{m}$.

transgenic mice were crossed with the Podocin-Cre driver line (49). The $Z / A P$ reporter line was from JAX and was maintained on a 129/BL6 background. The Kim $1^{\Delta m u c}$ has been described (34). The Z/AP and Z/Kim1-AP mice were genotyped using $\beta$-Geo-specific primers. The Six2-GC allele was genotyped as described (29). The Podocin-Cre allele was genotyped using Cre primers. In all cases, littermate control phenotypes were compared. Littermate controls were used for all mouse experiments.

Induction of renal fibrosis by UUO. Male BALB/c mice aged 8 to 10 weeks weighing 20-22 g were purchased from Charles River Laboratories. Mice were anesthetized and the left kidney exposed by flank incision. The ureter was ligated at 2 points proximal to the kidney with 6-0 silk. Sham animals had kidney exposed, but ureter was not tied.

Tissue preparation and histology. Mice were anesthetized, sacrificed, and immediately perfused via the left ventricle with ice-cold PBS for 2 minutes. Kidneys were hemi-sectioned, and portions were snap frozen in liquid nitrogen. Other kidneys were fixed in $10 \%$ neutral buffered formalin at $4^{\circ} \mathrm{C}$ for 12 hours, processed, embedded in paraffin wax, sectioned, and stained with PAS using standard procedures. Some kidneys were prepared for electron microscopy by glutaraldehyde fixation. Other kidneys were fixed in 4\% PLP fixative (4\% paraformaldehyde, $75 \mathrm{mM}$ L-lysine, $10 \mathrm{mM}$ sodium periodate) for 2 hours at $4{ }^{\circ} \mathrm{C}$, cryoprotected in $30 \%$ sucrose and snap frozen in OCT (Sakura FineTek). PAS-stained paraffin sections were assessed by quantitative measurement of tubular injury in 10 individual high-power fields (magnification, $\times 400$ ) per kidney. A percentage of the area affected was estimated for the number of necrotic cells, loss of brush border, cast formation, and tubule dilation and was scored as follows: $0,0 \%$ to $5 \% ; 1,5 \%$ to $10 \% ; 2,11 \%$ to $25 \% ; 3,26 \%$ to $45 \% ; 4,46 \%$ to $75 \%$, and 5 , more than $76 \%$. The fields analyzed in each section were selected at random, and evaluations were made in a blinded fashion.
Glomeruli were counted on PAS-stained sections from with the operator blinded to the genotype. Glomerular diameter was measured digitally, taking the average of glomerular width and height from individual glomeruli. The ventricular wall ratio was assessed by measuring both the dorsoventral and lateral ventricle diameters and dividing by the ventricular cavity diameter.

Immunofluorescence and immunohistochemical staining. Cryosections of $7-\mu \mathrm{m}$ were mounted on Fisher Superfrost Plus (Fisher) microscope slides, air dried, and treated for immunofluorescence as described (50). Primary antibodies against the following proteins were used: KIM-1 was detected with rabbit polyclonal anti-peptide antibody R9 exactly as described for both immunofluorescence and Western analysis (16); F4/80 (rat, 1:100, cat. no. 6640; Abcam); SMA (mouse FITC coupled, 1:200, cat. no. F3777, Sigma-Aldrich); DBA (lectin, 1:500, cat. no. L-1030; Vector Laboratories); Ki67 (rabbit monoclonal, 1:1000, cat. no. VP-RM04; Vector Laboratories); CD3 (rabbit monoclonal, 1:500 cat. no. VP-RM01; Vector Laboratories); and neutrophil (rat monoclonal, 1:100, cat. no. sc-71674; Santa Cruz Biotechnology Inc.). Secondary antibodies were obtained from Dako. Sections were mounted in Vectashield containing 4',6-diamino-2-phenylindole (Vector Labs). Images were taken with a Nikon TE2000 microscope CoolSnap camera (Roper Scientific) and processed using IP Lab Software (BD Biosciences). Immunofluorescence images were obtained on a Nikon TE2000 or a Nikon C1 D-Eclipse confocal microscope using standard procedures.

Immunohistochemical stains ( $\alpha$ SMA, CD3, neutrophil, KIM-1) were performed on formalin-fixed, paraffin-embedded $4-\mu \mathrm{M}$ sections. Sections were rehydrated and antigens retrieved using heated citrate. Staining was visualized using horseradish peroxidase-coupled secondary antibodies (Vectastain Elite; Vector Labs). LacZ activity was measured by standard $\mathrm{X}$-gal staining protocol on $7-\mu \mathrm{M}$ frozen kidney sections that had been fixed in PLP for 2 hours and was carried out for $12-24$ hours at $37^{\circ} \mathrm{C}$; then 
A

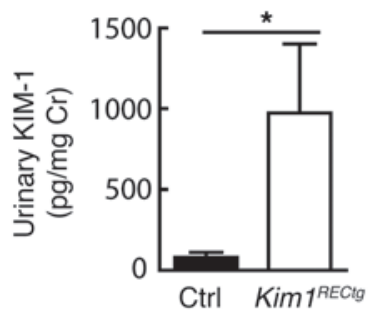

D

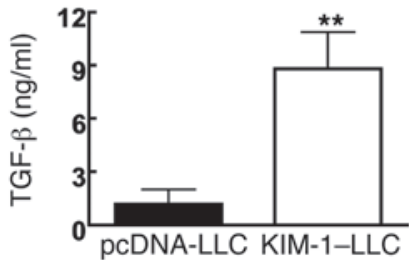

G

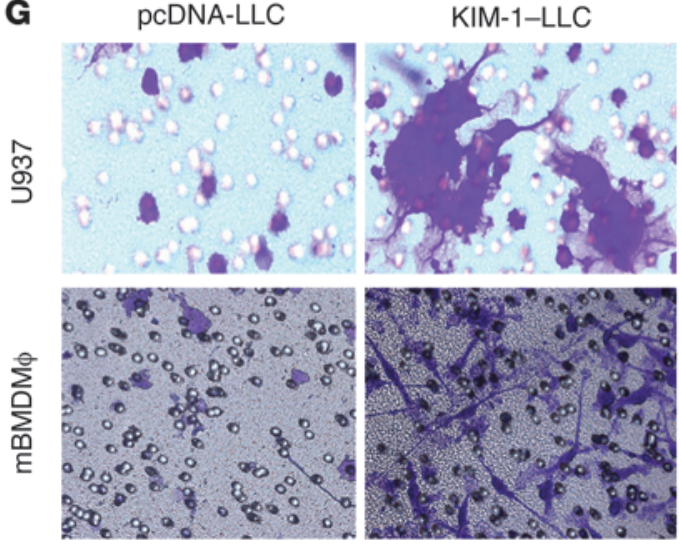

B

E
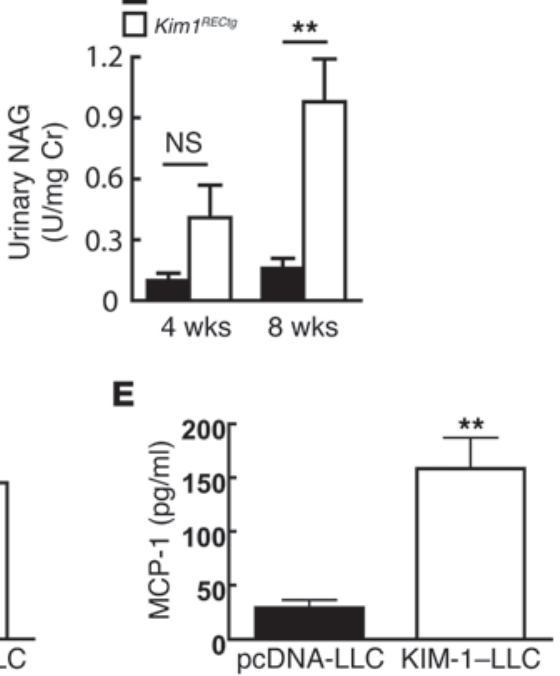

H

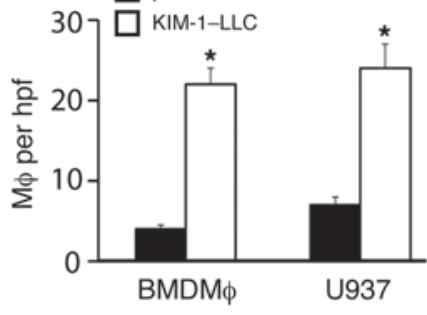

J

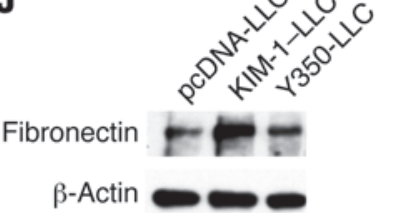

C

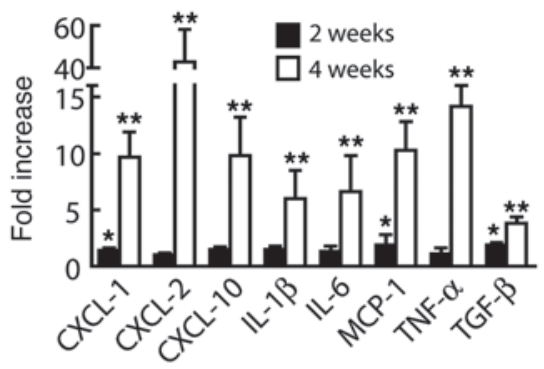

$\mathbf{F}$

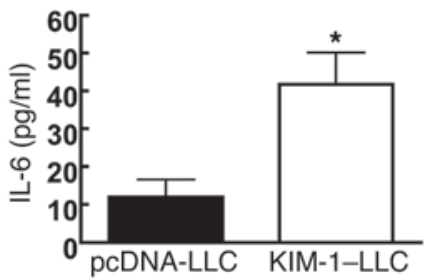

I

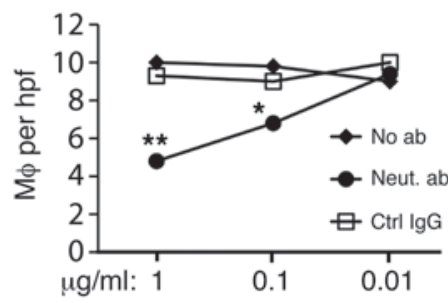

Figure 7

Persistent KIM-1 expression induces epithelial damage and proinflammatory cytokines in kidney epithelia in vivo and in vitro. (A) Shed KIM-1 is detectable in urine of Kim1 ${ }^{R E C t g}$ at 4 weeks of age compared with controls. ${ }^{*} P=0.03$. (B) Evidence of tubular injury in Kim $1^{R E C t g}$ is also reflected by increasing urinary NAG in 4- and 8-week-old Kim1RECtg. ${ }^{*} P<0.01$. (C) qPCR of kidney cortex cytokines, presented as fold increase in Kim1 1 RECtg $(n=3)$ compared with controls $(n=3)$ at 2 weeks (black bars) or 4 weeks of age (white bars). At 2 weeks, there is mild induction of CXCL-1, MCP-1, and TGF- $\beta$, with much higher levels of mRNA expression for all soluble cytokines by 4 weeks of age. ${ }^{*} P<0.05$; ${ }^{* *} P<0.006$. (D-F) Porcine proximal tubule epithelial cells stably transfected with either pcDNA3 control plasmid (pcDNA-LLC) or KIM-1 plasmid (KIM-1-LLC) spontaneously express TGF- $\beta$, MCP-1, and IL-6 ( $n=3$ for each). ${ }^{*} P<0.05 ;{ }^{* *} P<0.01$. (G and $\mathbf{H}$ ) Boyden chamber assay with pcDNA-LLC or KIM-1-LLC seeded on the bottom well and either U937 or mBMDM applied to top filter. Migration was measured after 3 hours. ${ }^{*} P<0.05$. Original magnification, $\times 500$. (I) Neutralizing antibody against MCP-1 abrogated KIM-1-LLC-dependent migration but not control IgG. ${ }^{\star} P<0.05 ;{ }^{\star \star} P<0.01$. (J) Cellular lysates from pcDNA-LLC, KIM-1-LLC, or LLC-PK1 cells transfected with KIM-1-Y350F (Y350-LLC) probed for fibronectin by Western blot demonstrate strong induction of fibronectin in KIM-1, but not Y350F-KIM-1-transfected cells.

sections were counterstained with eosin (Sigma-Aldrich) and mounted. PAS, H\&E, and Masson's trichrome stains were performed using standard techniques. AP activity was measured using NBT/BCPIP, with a standard protocol that included a 30 -minute incubation at $60^{\circ} \mathrm{C}$ to inactivate endogenous AP activity.

Histologic analysis. Histological analysis was performed on paraffin-embedded and serially cut kidney sections $(3 \mu \mathrm{m})$ stained with H\&E, PAS, and Masson's trichrome. Analysis of tubules included the evaluation of epithelial histology. The degree of injury was scored semiquantitatively on a 0 to 4 scale for reabsorption granules, vacuolization, and epithelial degeneration as follows: 0 , no lesion; 1 , minimal (minor focal changes); 2 , mild; 3 , moderate; 4 , severe. The distribution was graded as focal if $49 \%$ or less and dif- fuse if $50 \%$ or more of the tubule showed these changes. Semiquantitative analysis of glomeruli included glomerular histology as well as foot process morphology assessed by electron microscopy and was graded as follows: minimal: 1 (involving < $5 \%$ of glomerulus); mild: 2 (5\%-24\%); moderate: 3 (25\%-49\%); and severe: 4 ( $\geq 50 \%$ ). For assessment of glomerular involvement, an average of $80-120$ glomeruli per section were examined on multiple levels. All scoring was done in a blinded manner by an experienced renal pathologist. Semiquantitative analysis of tubular morphology in Kim $1^{\Delta m u c}$ mice was also performed in a blinded fashion exactly as described (51).

Electron microscopy. Portions of kidneys were fixed in Karnovsky's fixative and processed for electron microscopic studies by standard procedures. Semithin sections of each block were stained with toluidine blue 
A

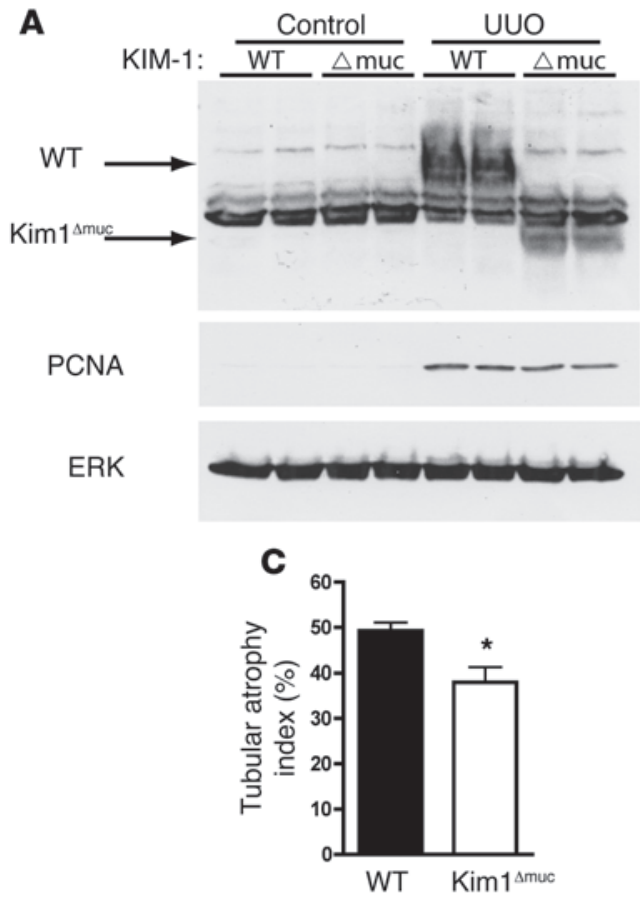

B Control (Trichrome)

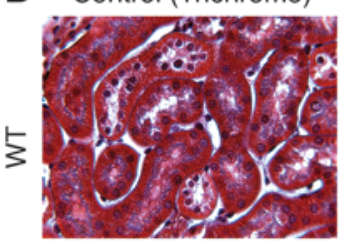

$-56 \mathrm{kDa}$

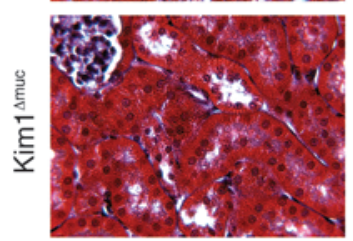

UUO (Trichrome)
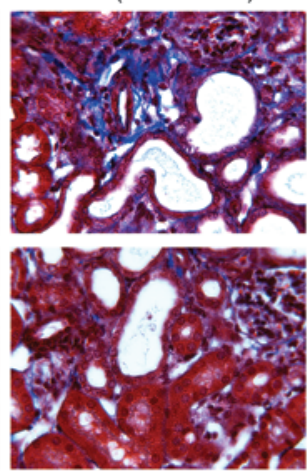

UUO (PAS)
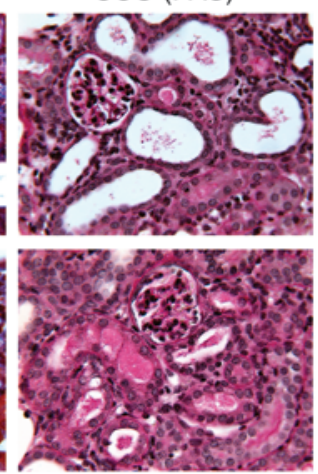

D

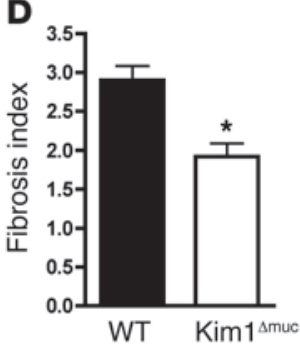

G Fibronectin

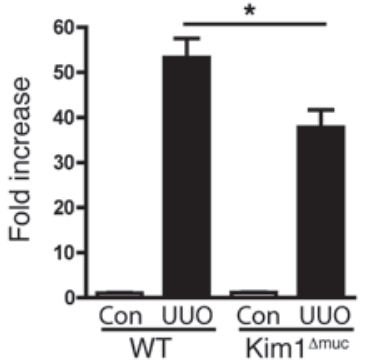

E

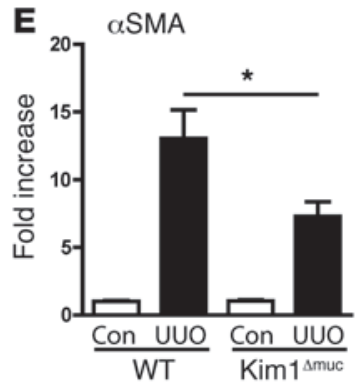

H MCP-1

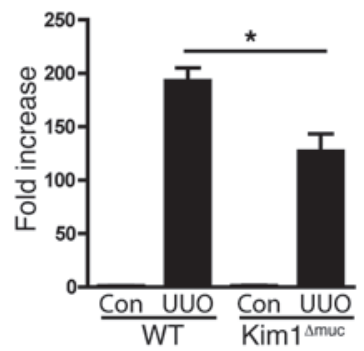

Figure 8

The functional mutant Kim1 ${ }^{\Delta m u c}$ is protected from kidney fibrosis. (A) Control and Kim1 ${ }^{\Delta m u c}$ mice were subjected to UUO and sacrificed at day 2. Kidney lysates reveal KIM-1 protein at $75 \mathrm{kDa}$ in the control, but at $55 \mathrm{kDa}$ in the Kim1 ${ }^{\Delta m u c}$, corresponding to the deletion of exon 3 (arrows). The arrowhead identifies a nonspecific Ig band. Proliferating cell nuclear antigen (PCNA) staining reflects increased cell proliferation after UUO. (B) Control or Kim1 ${ }^{\Delta m u c}$ kidney sections before or after UUO. There is reduced interstitial collagen in Kim1 ${ }^{\Delta m u c}$ reflected by Masson's trichrome stain, and reduced tubular injury (PAS). (C and D) Quantification of tubular atrophy and fibrosis index, respectively ( $n=5$ kidneys each condition). (E-G) qPCR of kidney cortex fibrosis, presented as fold increase of $\operatorname{Kim} 1^{\text {RECtg }}(n=5)$ compared with control $(n=5)$ at 10 days after UUO. ${ }^{*} P<0.05$. (H) Reduced MCP-1 mRNA by qPCR in Kim1 ${ }^{\Delta m u c}$ compared with control $(n=5) .{ }^{*} P<0.05$.

stain and examined by light microscopy to select for ultrathin sectioning. Ultrathin sections were stained with uranyl acetate and lead citrate and examined by electron microscopy.

Western blot analysis. Kidney tissues were lysed, and lysates were prepared as previously described (50). Membranes were incubated with 1 or more of the following primary antibodies: rabbit antibody to LacZ ( 1 in 5,000; Cappel), chicken antibody to GFP ( 1 in 1,000 ; AVES), rabbit antibody to KIM-1 ( 1 in 250) (12), rabbit anti-fibronectin ( 1 in 1,000; Abcam), proliferating nuclear cell antigen ( 1 in 1,000; Abcam) and ERK ( 1 in 1,000; Cell Signaling). Horseradish peroxidase-conjugated secondary antibodies were applied, and enhanced chemiluminescence (Amersham Biosciences) was used to detect proteins.

Quantification of $m R N A$ by reverse transcription PCR. Total RNA was isolated from snap-frozen kidneys with RNeasy columns (QIAGEN). Five micrograms of total RNA was treated with DNAse I (Invitrogen) and reverse transcribed with the M-MLV reverse transcriptase kit and Oligo dT primers (Promega). Real-time PCR was performed by TaqMan gene expression assays (Applied Biosystems) for detection of mRNA expression using GAPDH as the internal control. Qualitative RT-PCR was performed on 1/200th of the RT product using the following primer pairs (from $5^{\prime}$ to $3^{\prime}$ ): Cre forward: TTCCCGCAGAACCTGAAGATG, reverse: CCCCAGAAATGCCAGATTACG; KIM-1 forward: ATGAATCAGATTCAAGTCTTC, reverse: TCTGGTTTGTGAGTCCATGTG; GAPDH forward: TGGAGAAACCTGCCAAGTA, reverse: AAGAGTGGGAGTTGCTGTTG. The cycling conditions were as follows: Cre: melting temperature $(\mathrm{Tm}) 57^{\circ} \mathrm{C}$ and 35 cycles, KIM-1: $\mathrm{Tm} 55^{\circ} \mathrm{C}$ and 30 cycles; GAPDH: $\operatorname{Tm} 53^{\circ} \mathrm{C}$ and 25 cycles. PCR products were visualized on ethidium bromide containing, $2 \%$ agarose gels and photographed.

Quantitative PCR was performed using a Bio-Rad iCycler and the following primers (from $5^{\prime}$ to $3^{\prime}$ ): Col-1 $\alpha 1$ forward: TGACTGGAAGAGCGGAGAGT, reverse: GTTCGGGCTGATGTACCAGT; CXCL1 
forward: CTGGGATTCACCTCAAGAACATC, reverse: CAGGGTCAAGGCAAGCCTC; CXCL2 forward: CCAACCACCAGGCTACAGG, reverse: GCGTCACACTCAAGCTCTG; CXCL10 forward: CCAAGTGCTGCCGTCATTTTC, reverse: GGCTCGCAGGGATGATTTCAA; GAPDH forward: CATGTTCCAGTATGACTCCACTC, R: GGCCTCACCCCATTTGATGT; IL-1 $\beta$ forward: CCTTCCAGGATGAGGACATGA, reverse: AACGTCACACACCAGCAGGTT; IL-6 forward: TAGTCCTTCCTACCСCAATTTCC, reverse: TTGGTCCTTAGCCACTCCTTC; MCP-1 forward: TGCATCTGCCCTAAGGTCTTC, reverse: AAGTGCTTGAGGTGGTTGTGG; TGF- $\beta$ forward: GCAACAATTCCTGGCGTTACC, reverse: CGAAAGCCCTGTATTCCGTCT; TNF- $\alpha$ forward: CCCTCACACTCAGATCATCTTCT, reverse: GCTACGACGTGGGCTACAG; $\alpha$ SMA forward: CTGACAGAGGCACCACTGAA, reverse: CATCTCCAGAGTCCAGCACA; fibronectin forward: ATGTGGACCCCTCCTGATAGT, R: GCCCAGTGATTTCAGCAAAGG.

Physiologic measurements. Serum creatinine was measured using a Beckman Creatinine Analyzer 2 by the Jaffe rate method. Hematocrit was calculated after centrifugation of a hematocrit capillary tube. Proteinuria was assessed by the micro pyrogallol red method (total protein kit; Sigma-Aldrich) or by separation of $1 \mu \mathrm{l}$ of urine on a 10\% SDS-PAGE gel followed by coomassie stain. Blood pressure was assessed by tail-cuff analyzer (Visitech Systems Inc.; Apex). After training conscious mice for 3 days, systolic blood pressure and heart rate were collected for 3 consecutive days between 9 am and $11 \mathrm{am}$, with an average of 10 reads each day. KIM-1 protein in urine was measured by microsphere-based Luminex technology. NAG in urine was measured by colorimetric assayed using a commercial kit (Roche). For serum electrolytes, $\mathrm{BUN}$, and creatinine, mice were anesthetized and the blood samples were collected from carotid artery. Analyses were assessed by a core laboratory (Children's Hospital Boston, Boston, Massachusetts, USA).

Cell culture. Cos7 cells were transfected using Lipofectamine 2000 (Invitrogen). Porcine proximal tubular epithelial cells (LLC-PK1) cells were grown in DMEM supplemented with $10 \% \mathrm{FBS}$ and maintained at $37^{\circ} \mathrm{C}$ in $5 \%$ $\mathrm{CO}_{2}$. To create stable cell lines expressing full-length KIM-1 (KIM-1-LLC), LLC-PK1 cells were transfected with pcDNA3-KIM-1 or pcDNA3-KIM-1Y350F plasmid and the stable population was selected using G418 treatment (400 $\mu \mathrm{g} / \mathrm{ml}$ ). Control cell lines (pcDNA-LLC) stably expressing pcDNA3-neo were generated the same way. To generate $\mathrm{MBMDM}$, femurs and tibias were removed from 20- to 25-g BALB/c mice. BM was isolated from these by standard sterile techniques and matured for 7 days in uncoated Petri dishes using DMEM/F12 medium with $10 \%$ FCS, penicillin $(100 \mathrm{U} / \mathrm{ml})$, and streptomycin $(100 \mathrm{mg} / \mathrm{ml})$ and conditioned with M-CSF from L929 cells. The U937 cell line, a human monomyelocytic cell line, was cultured in RPMI 1640 medium supplemented with $10 \% \mathrm{FCS}, 1 \%$ penicillin/streptomycin, and $2 \mathrm{mM} \mathrm{L-gluta-}$ mine. Cells were subcultured 3 times a week and maintained at a concentration of $0.5-1.0 \times 10^{6}$ cells $/ \mathrm{ml}$. Monocytic differentiation of U937 cells was achieved by adding $10 \mathrm{nM}$ PMA for 48 hours. PMA-differentiated U937 was washed 3 times by sterile PBS before experiments.

Cytokine measurement. Both KIM-1-LLC and pcDNA-LLC cells were grown to confluence, and supernatant was collected, centrifuged, and stored at $-80^{\circ} \mathrm{C}$ until further analysis. Alternatively, mouse urine was col- lected with a metabolic cage and processed in a similar fashion. MCP-1, IL- 6 , and TGF- $\beta$ microbead-based assays were developed and validated in the lab. Approximately 6000 beads $/ 50 \mu \mathrm{l}$ were incubated with $30 \mu \mathrm{l}$ of sample or recombinant proteins (R\&D Systems) for 1 hour, washed 3 times with PBST, and incubated in corresponding biotinylated antibodies (R\&D Systems) for 45 minutes on an orbital shaker at $300 \mathrm{rpm}$. Beads were washed again with PBST and incubated for 15 minutes with streptavidin-PE solution (Invitrogen). The signal from the fluorochrome, which is directly proportional to the amount of antigen bound at the micro-bead surface, ws captured using the Bio-Plex 200 system (Bio-Rad). Data were generated and interpreted using parametric logistic regression analysis.

Boyden chamber assay. Cell chemotaxis assay was performed in a modified Boyden chamber using 24-well flat-bottom tissue plates with $5-\mu \mathrm{m}$ polyethylenterephtylan membrane inserts (BD). The lower compartment of each chamber was filled with $500 \mu \mathrm{l}$ of the conditioned medium. Membrane inserts were filled with $300 \mu \mathrm{l}$ of cell suspension and placed in the prefilled lower compartments. The chambers were then incubated for 3 hours in $37^{\circ} \mathrm{C}, 5 \% \mathrm{CO}_{2}$. After incubation, nonmigrated cells in the upper wells were removed by scraping and the migrated cells were stained with eosin on the membrane. Adherent cells on the lower surface of the membrane were counted from 5 high power fields by light microscopy $(\times 40)$. Data are presented as cells per high-power field. Neutralizing antibody against MCP-1 was from Sigma-Aldrich.

Statistics. All results are reported as mean \pm SEM. All error bars on graphs represent SEM. Statistical tests are 2 -tailed, unpaired $t$ tests except for survival analysis (Figure 4E), which used the log rank test, and Figures 7B and Supplemental Figure 2, which used a repeated measures $t$ test.

Study approval. All animal studies were approved by the Harvard Institutional Animal Care and Use Committee.

\section{Acknowledgments}

We thank Corrine Lobe for the Z/AP plasmid and Jordan Kriedberg for the Podocin-Cre transgenic mice. This work was supported by NIH grants DK73628, DK84316, DK088923, funds from the Harvard Stem Cell Institute, and an Established Investigator Award from the American Heart Association to B.D. Humphreys and DK39773 and DK72381 to J.V. Bonventre. Work in A.P. McMahon's laboratory is supported by the National Institute of Diabetes and Digestive and Kidney Diseases (NIDDK). N. Wang was supported by the China Scholarship Council-Harvard University Exchange. S. Xiao was supported by NIDDK K01 DK090105. I. Grgic was supported by a fellowship of the Deutsche Forschungsgemeinschaft.

Received for publication July 25, 2012, and accepted in revised form June 17, 2013.

Address correspondence to: Benjamin D. Humphreys, Brigham and Women's Hospital, Harvard Institutes of Medicine, Rm 550, 4 Blackfan Circle, Boston, Massachusetts 02115, USA. Phone: 617.525.5971; Fax: 617.525.5965; E-mail: bhumphreys@partners.org.
1. Clarkson MR, Friedewald JJ, Eustace H, Rabb H. Acute kidney injury. In: Brenner BM, ed. Brenner and Rector's The Kidney. 8th ed. Philadelphia, Pennsylvania, USA: Saunders Elsevier; 2008:943-986.

2. Ishani A, et al. Acute kidney injury increases risk of ESRD among elderly. J Am Soc Nephrol. 2009; 20(1):223-228.

3. Wald $\mathrm{R}$, et al. Chronic dialysis and death among survivors of acute kidney injury requiring dialysis. JAMA. 2009;302(11):1179-1185.

4. Lafrance JP, Miller DR. Acute kidney injury associates with increased long-term mortality. J Am Soc
Nephrol. 2010;21(2):345-352.

5. Hsu CY, McCulloch CE, Fan D, Ordoñez JD, Chertow GM, Go AS. Community-based incidence of acute renal failure. Kidney Int. 2007;72(2):208-212.

6. Waikar SS, Curhan GC, Wald R, McCarthy EP, Chertow GM. Declining mortality in patients with acute renal failure, 1988 to 2002. J Am Soc Nephrol. 2006; 17(4):1143-1150.

7. Hsu CY. Linking the population epidemiology of acute renal failure, chronic kidney disease and endstage renal disease. Curr Opin Nephrol Hypertens. 2007;16(3):221-226.
8. Basile DP, Donohoe D, Roethe K, Osborn JL. Renal ischemic injury results in permanent damage to peritubular capillaries and influences longterm function. Am J Physiol Renal Physiol. 2001; 281(5):F887-F899.

9. Kang DH, et al. Role of the microvascular endothelium in progressive renal disease. J Am Soc Nephrol. 2002;13(3):806-816.

10. Nangaku M. Chronic hypoxia and tubulointerstitial injury: a final common pathway to end-stage renal failure. J Am Soc Nephrol. 2006;17(1):17-25.

11. Rennert PD. Novel roles for TIM-1 in immunity 
and infection. Immunol Lett. 2011;141(1):28-35.

12. Ichimura $T$, et al. Kidney injury molecule-1 (KIM-1), a putative epithelial cell adhesion molecule containing a novel immunoglobulin domain, is up-regulated in renal cells after injury. J Biol Chem. 1998; 273(7):4135-4142.

13. Kobayashi N, et al. TIM-1 and TIM-4 glycoproteins bind phosphatidylserine and mediate uptake of apoptotic cells. Immunity. 2007;27(6):927-940.

14. Miyanishi M, Tada K, Koike M, Uchiyama Y, Kitamura T, Nagata S. Identification of Tim4 as a phosphatidylserine receptor. Nature. 2007; 450(7168):435-439.

15. Ichimura T, Asseldonk EJ, Humphreys BD, Gunaratnam L, Duffield JS, Bonventre JV. Kidney injury molecule- 1 is a phosphatidylserine receptor that confers a phagocytic phenotype on epithelial cells. J Clin Invest. 2008;118(5):1657-1668.

16. Kuehn EW, Park KM, Somlo S, Bonventre JV. Kidney injury molecule- 1 expression in murine polycystic kidney disease. Am J Physiol Renal Physiol. 2002; 283(6):F1326-F1336.

17. Perez-Rojas J, et al. Mineralocorticoid receptor blockade confers renoprotection in preexisting chronic cyclosporine nephrotoxicity. Am J Physiol Renal Physiol. 2007;292(1):F131-F139.

18. van Timmeren MM, et al. Tubular kidney injury molecule-1 in protein-overload nephropathy. AmJ Physiol Renal Physiol. 2006;291(2):F456-F464.

19. Kramer AB, et al. Reduction of proteinuria in adriamycin-induced nephropathy is associated with reduction of renal Kidney injury molecule-1 (Kim-1) over time. Am J Physiol Renal Physiol. 2009; 296(5):F1136-F1145

20. Waanders F, van Timmeren MM, Stegeman CA, Bakker SJ, van Goor H. Kidney injury molecule-1 in renal disease. J Pathol. 2010;220(1):7-16.

21. van Timmeren MM, van den Heuvel MC, Bailly V, Bakker SJ, van Goor H, Stegeman CA. Tubular kidney injury molecule-1 (KIM-1) in human renal disease. J Pathol. 2007;212(2):209-217.

22. Schroppel B, et al. Tubular expression of KIM-1 does not predict delayed function after transplantation. J Am Soc Nephrol. 2010;21(3):536-542.

23. Waanders F, et al. Effect of renin-angiotensin-aldosterone system inhibition, dietary sodium restriction, and/or diuretics on urinary kidney injury molecule 1 excretion in nondiabetic proteinuric kidney disease: a post hoc analysis of a randomized controlled trial. Am J Kidney Dis. 2009;53(1):16-25.

24. van Timmeren MM, et al. High urinary excretion of kidney injury molecule- 1 is an independent pre- dictor of graft loss in renal transplant recipients. Transplantation. 2007;84(12):1625-1630.

25. Okusa MD, Chertow GM, Portilla D. The nexus of acute kidney injury, chronic kidney disease, and World Kidney Day 2009. Clin J Am Soc Nephrol. 2009; 4(3):520-522.

26. Rees AJ, Kain R. Kim-1/Tim-1: from biomarker to therapeutic target? Nephrol Dial Transplant. 2008; 23(11):3394-3396.

27. Kobayashi A, et al. Six 2 defines and regulates a multipotent self-renewing nephron progenitor population throughout mammalian kidney development. Cell Stem Cell. 2008;3(2):169-181.

28. Humphreys BD, et al. Fate tracing reveals the pericyte and not epithelial origin of myofibroblasts in kidney fibrosis. Am J Pathol. 2010;176(1):85-97.

29. Humphreys BD, et al. Intrinsic epithelial cells repair the kidney after injury. Cell Stem Cell. 2008; 2(3):284-291.

30. Ritz E. Left ventricular hypertrophy in renal disease: beyond preload and afterload. Kidney Int. 2009; 75(8):771-773.

31. Guo JK, et al. The commonly used beta-actinGFP transgenic mouse strain develops a distinct type of glomerulosclerosis. Transgenic Res. 2007; 16(6):829-834.

32. Yamanishi Y, et al. TIM1 is an endogenous ligand for LMIR5/CD300b: LMIR5 deficiency ameliorates mouse kidney ischemia/reperfusion injury. J Exp Med. 2010;207(7):1501-1511.

33. Ren L, et al. Soluble fibronectin induces chemokine gene expression in renal tubular epithelial cells. Kidney Int. 2005;68(5):2111-2120.

34. Xiao S, et al. Defect in regulatory B-cell function and development of systemic autoimmunity in T-cell Ig mucin 1 (Tim-1) mucin domain-mutant mice. Proc Natl Acad Sci U S A. 2012;109(30):12105-12110.

35. Yang L, Besschetnova TY, Brooks CR, Shah JV, Bonventre JV. Epithelial cell cycle arrest in G2/M mediates kidney fibrosis after injury. Nat Med. 2010; 16(5):535-543.

36. Grgic I, et al. Targeted proximal tubule injury triggers interstitial fibrosis and glomerulosclerosis. Kidney Int. 2012;82(2):172-183.

37. Sizing ID, et al. Epitope-dependent effect of antimurine TIM-1 monoclonal antibodies on T cell activity and lung immune responses. J Immunol. 2007;178(4):2249-2261

38. Fukushima A, et al. Antibodies to T-cell Ig and mucin domain-containing proteins (Tim) -1 and -3 suppress the induction and progression of murine allergic conjunctivitis. Biochem Biophys Res Commun.
2007;353(1):211-216

39. Feng BS, et al. Disruption of T-cell immunoglobulin and mucin domain molecule (TIM)-1/TIM4 interaction as a therapeutic strategy in a dendritic cell-induced peanut allergy model. J Allergy Clin Immunol. 2008;122(1):55-61.

40. Sonar SS, et al. Antagonism of TIM-1 blocks the development of disease in a humanized mouse model of allergic asthma. J Clin Invest. 2010; 120(8):2767-2781.

41. Brenner BM, Garcia DL, Anderson S. Glomeruli and blood pressure. Less of one, more the other? Am J Hypertens. 1988;1(4 pt 1):335-347.

42. Dziarmaga A, Eccles M, Goodyer P. Suppression of ureteric bud apoptosis rescues nephron endowment and adult renal function in Pax2 mutant mice. J Am Soc Nephrol. 2006;17(6):1568-1575.

43. Wintour EM, Moritz KM, Johnson K, Ricardo S, Samuel CS, Dodic M. Reduced nephron number in adult sheep, hypertensive as a result of prenatal glucocorticoid treatment. J Physiol. 2003;549(pt 3):929-935.

44. Moritz KM, Jefferies A, Wong J, Wintour EM, Dodic M. Reduced renal reserve and increased cardiac output in adult female sheep uninephrectomized as fetuses. Kidney Int. 2005;67(3):822-828.

45. Vehaskari VM, Aviles DH, Manning J. Prenatal programming of adult hypertension in the rat. Kidney Int. 2001;59(1):238-245.

46. Kitagawa K, et al. Blockade of CCR2 ameliorates progressive fibrosis in kidney. Am J Pathol. 2004; 165(1):237-246.

47. Lucas M, Stuart LM, Savill J, Lacy-Hulbert A. Apoptotic cells and innate immune stimuli combine to regulate macrophage cytokine secretion. Jimmunol. 2003;171(5):2610-2615.

48. Lobe CG, Koop KE, Kreppner W, Lomeli H, Gertsenstein M, Nagy A. Z/AP, a double reporter for cre-mediated recombination. Dev Biol. 1999; 208(2):281-292.

49. Ho J, Ng KH, Rosen S, Dostal A, Gregory RI, Kreidberg JA. Podocyte-specific loss of functional microRNAs leads to rapid glomerular and tubular injury. J Am Soc Nephrol. 2008;19(11):2069-2075.

50. Park KM, Kramers C, Vayssier-Taussat M, Chen A, Bonventre JV. Prevention of kidney ischemia/reperfusion-induced functional injury, MAPK and MAPK kinase activation, and inflammation by remote transient ureteral obstruction. J Biol Chem. 2002; 277(3):2040-2049.

51. Sugimoto $\mathrm{H}$, et al. Activin-like kinase 3 is important for kidney regeneration and reversal of fibrosis. Nat Med. 2012;18(3):396-404. 